ESAIM: M2AN

Vol. 41, No 4, 2007, pp. 743-769

DOI: $10.1051 / \mathrm{m} 2 \mathrm{an}: 2007039$
ESAIM: Mathematical Modelling and Numerical Analysis

www.esaim-m2an.org

\title{
ON THE STABILITY OF THE COUPLING OF 3D AND 1D FLUID-STRUCTURE INTERACTION MODELS FOR BLOOD FLOW SIMULATIONS
}

\author{
Luca Formaggia $^{1}$, Alexandra Moura $^{1}$ and Fabio Nobile ${ }^{1}$
}

\begin{abstract}
We consider the coupling between three-dimensional (3D) and one-dimensional (1D) fluidstructure interaction (FSI) models describing blood flow inside compliant vessels. The 1D model is a hyperbolic system of partial differential equations. The 3D model consists of the Navier-Stokes equations for incompressible Newtonian fluids coupled with a model for the vessel wall dynamics. A non standard formulation for the Navier-Stokes equations is adopted to have suitable boundary conditions for the coupling of the models. With this we derive an energy estimate for the fully 3D$1 \mathrm{D}$ FSI coupling. We consider several possible models for the mechanics of the vessel wall in the 3D problem and show how the 3D-1D coupling depends on them. Several comparative numerical tests illustrating the coupling are presented.
\end{abstract}

Mathematics Subject Classification. 65M12, 65M60, 92C50, 74F10, 76Z05.

Received November 15, 2006. Revised March 19, 2007.

\section{INTRODUCTION}

Blood flow in large arteries is characterized by traveling pressure waves, also called pulse waves, which are due to the interaction between the blood and the artery wall, which deforms under the action of the fluid pressure $[26,31]$. A possible mathematical model describing such phenomena consists of the 3D Navier-Stokes equations for incompressible Newtonian fluids, since blood is considered incompressible and obeys a Newtonian rheology in medium to large vessels (see $[21,34]$ ). These equations are coupled with a model describing the movement of the wall, which can not be neglected in large vessels since deformations up to $10 \%$ of the vessel radius may occur during the cardiac cycle. The numerical simulation of the pulse propagation phenomena induced by this coupling is extremely challenging [36]. In particular, the large added mass effect on the structure greatly increases the computational costs, making it difficult to simulate large regions [4]. Although significant advances have been made during the last years on the efficiency of fully implicit $[11,19,20]$ or semi-implicit $[12,29,35]$ algorithms for fluid-structure interaction, and by now simulations of rather large arterial trees, like those in [3, 41], are possible, their computational cost still discourage an extensive usage. Furthermore, often one is interested in the detailed flow field only on a limited region, like a particular artery or a major bifurcation. Yet, the human

Keywords and phrases. Fluid-structure interaction, 3D-1D FSI coupling, energy estimate, multiscale models.

1 MOX - Modelling and Scientific Computing, Department of Mathematics, Politecnico di Milano, Italy.

luca.formaggia@polimi.it; alexandra.moura@polimi.it; fabio.nobile@polimi.it

(C) EDP Sciences, SMAI 2007 
cardiovascular system is formed by a closed network of vessels with a high level of interdependence and realistic numerical simulations cannot be fully accomplished without taking this into account $[17,36]$.

This motivates the adoption of simplified models, like for instance 1D models, originally proposed by Euler [10] and widely used nowadays $[32,39]$. These models are described by hyperbolic systems of partial differential equations and, despite having a lower level of accuracy compared to the fully 3D model, they capture effectively the pulse waves at a much lower computational cost. This allows one to properly simulate pressure waves propagation in large regions of the arterial tree by a network of these models coupled together [16-18, 36].

Still, the need of detailed information calls for a more complex description in specific sub-regions of interest. In fact, it is widely accepted that local blood flow is strongly related to the initiation and progression of cardiovascular diseases, like atherosclerosis [3,28]. The information needed for these investigations, like wall shear stress or local velocity patterns, may be computed only resorting to $3 \mathrm{D}$ simulations. By coupling the detailed 3D FSI model with the reduced 1D it is possible to combine both requirements of having detailed information locally while accounting for the global circulation. From another perspective, the 1D network model can be thought as an absorbing (or far field) boundary condition for the 3D detailed model.

This coupling of different models is known as the geometrical multiscale approach [13] and may also include, besides 3D and 1D models, lumped parameters models [14,38]. Here we focus on the coupling between 3D and 1D FSI models. Very little can be found in literature regarding the properties of this coupling. In several applicative works, reduced models are coupled with 3D ones with the perspective of providing more realistic boundary conditions $[30,41]$, and not with the objective of embedding the 3D model into a larger arterial tree. Furthermore, in most of these applications the 3D model is assumed to be rigid and the reduced models are lumped parameters ones [25,41]. The work [9] proposed some coupling strategies and algorithms based on subdomain iterations, and analyzed the stability properties of each subproblem. However, a stability analysis of the fully $3 \mathrm{D}-1 \mathrm{D}$ coupled problem is not yet available.

In this work we address for the first time the analysis of the two models together. We propose a coupling based on the continuity, at the coupling interface, of the mass fluxes and normal component of the total stress. We adopt a proper reformulation of the Navier-Stokes equations, for which the natural boundary condition of the weak problem is precisely the normal total stress. With this choice of coupling conditions, and by means of the reformulation of the fluid equations, we are able to obtain an a priori energy estimate for the fully 3D-1D FSI problem. At the best of our knowledge, this result is new.

We also consider several possibilities for the structure model on the 3D problem and discuss in each case the stability of the coupling. Furthermore, we propose a numerical coupling algorithm, based on an explicit technique, and present several numerical tests. The present paper is presented in the context of blood flow in arteries, nevertheless the procedures here adopted may be extended to other similar problems where wave propagation phenomena arise due to a fluid-structure interaction in pipes.

The outline of the paper is as follows. In Section 2 we present the Navier-Stokes equations reformulated to have the normal total stresses as natural boundary condition. Being a non standard formulation, we carry out a well posedness analysis for the rigid wall case, providing an existence and unicity result for the steady case, under suitable hypothesis on the data.

In Section 3 we consider the motion of the structure wall, by introducing several possible models for the vessel wall dynamics, ranging from a simple 2D algebraic model to $3 \mathrm{D}$ non linear elasticity. We study the coupling between the adopted formulation of the fluid equations and the different models for the movement of the wall, and we derive an energy estimate in each case.

In Section 4 we recall the 1D model and its energy estimate, as derived in [9].

Section 5 is devoted to the full 3D-1D FSI coupling. Due to the formulation adopted for the Navier-Stokes equations, and by imposing the continuity of the fluxes and the normal total stresses, we are able to derive an energy estimate for the fully coupled model. We also discuss the dependence of that energy balance on the structure model chosen on the 3D side.

Numerical results are presented in Section 6. We propose an explicit numerical algorithm for the 3D-1D coupling, while an implicit coupling is applied on the 3D FSI problem and the Lax-Wendroff scheme is used for 


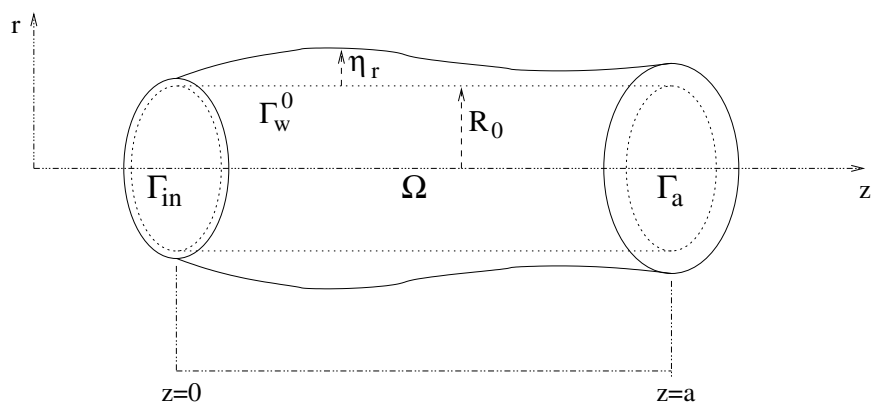

Figure 1. Cylindrical vascular region $\Omega^{t}$.

the 1D model. Several numerical tests are carried out, illustrating different coupling strategies. To validate the results, the coupling problem solution is compared to the solution of a complete 3D FSI problem in a longer domain.

\section{The Fluid EQuations}

Let $\Omega$ be a bounded domain of $\mathbb{R}^{3}$ representing a portion of a cylindrical artery. We denote by $\Gamma_{w}$ the portion of the boundary corresponding to the physical arterial wall, while $\Gamma_{i n}$ and $\Gamma_{a}$ represent the so called artificial boundaries, since they do not correspond to any physical interface (see Fig. 1). More precisely, $\Gamma_{i n}$ is the upstream or proximal section, closest to the heart, and $\Gamma_{a}$ is the downstream or distal section, closest to the peripheral vessels. We assume that the domain $\Omega \subset \mathbb{R}^{3}$ is open and connected and its boundary $\partial \Omega=\Gamma_{w} \cup \Gamma_{i n} \cup \Gamma_{a}$ is locally Lipschitz (i.e., $\left.\partial \Omega \in \mathcal{C}^{1,1}\right)$.

Using the vectorial identity

$$
\mathbf{u} \cdot \nabla \mathbf{u}=\frac{1}{2} \nabla|\mathbf{u}|^{2}+\operatorname{curl} \mathbf{u} \times \mathbf{u},
$$

we write the Navier-Stokes equations in the following form, in $\Omega$ and for all $t \in I=(0, T]$

$$
\left\{\begin{aligned}
\rho \frac{\partial \mathbf{u}}{\partial t}+\rho\left(\frac{1}{2} \nabla|\mathbf{u}|^{2}+\operatorname{curl} \mathbf{u} \times \mathbf{u}\right)+\nabla p-\operatorname{div}(2 \nu \mathbf{D}(\mathbf{u})) & =\mathbf{0} \\
\operatorname{div} \mathbf{u} & =0
\end{aligned}\right.
$$

where the unknowns are the fluid velocity $\mathbf{u}$ and pressure $p$. Furthermore, $\rho$ and $\nu>0$ are the density and the dynamic viscosity of the fluid, respectively, and are assumed to be constant; $\mathbf{D}$ is the strain tensor given by

$$
\mathbf{D}(\mathbf{u})=\frac{1}{2}\left(\nabla \mathbf{u}+\nabla^{T} \mathbf{u}\right)
$$

The choice of the rather unusual formulation (1) is motivated by the fact that the normal total stress becomes a natural boundary condition (hereafter also called Neumann boundary condition) once problem (1) is written in a weak form, as it will be shown next. On the other hand, we are interested in imposing the normal total stress at the downstream section in view of the coupling with the 1D model since, as we will show in Section 5 , this choice allow us to obtain a stable coupling.

We endow equations (1) with the initial condition

$$
\mathbf{u}=\mathbf{u}_{\mathbf{0}} \quad \text { for } t=0, \text { in } \Omega,
$$

and either Dirichlet boundary conditions

$$
\mathbf{u}=\mathbf{h}
$$


or Neumann boundary conditions

$$
\boldsymbol{\sigma}^{t o t}(\mathbf{u}, p) \cdot \mathbf{n}=\mathbf{q}
$$

on the artificial sections $\Gamma_{i n}$ and $\Gamma_{a}$, where $\mathbf{h}$ and $\mathbf{q}$ are given and $\boldsymbol{\sigma}^{\text {tot }}$ is the total stress tensor given by

$$
\boldsymbol{\sigma}^{t o t}(\mathbf{u}, p)=-\left(p+\frac{\rho}{2}|\mathbf{u}|^{2}\right) \mathbf{I}+2 \nu \mathbf{D}(\mathbf{u})
$$

We assume that, at any time $t \in I, \mathbf{h} \in\left[H^{1 / 2}(\gamma)\right]^{3}$ and $\mathbf{q} \in\left[H^{-1 / 2}(\gamma)\right]^{3}$, where $\gamma$ is $\Gamma_{i n}$ or $\Gamma_{a}$, depending on where the Dirichlet or Neumann boundary conditions, respectively, are being imposed.

At the boundary $\Gamma_{w}$ we take no-slip conditions, i.e.

$$
\mathbf{u}=\mathbf{g} \quad \text { for } t \in I, \text { on } \Gamma_{w}
$$

If the domain is fixed for all $t \in I$, then the no-slip condition is given by $\mathbf{g}=\mathbf{0}$. If a model for the wall dynamics is considered instead, and the domain changes in time, then the function $\mathbf{g}$ is the velocity of the structure wall, i.e. the velocity of the domain boundary.

Since the formulation of the Navier-Stokes equations we are adopting is a non standard one, we will first carry out a well posedness analysis of the problem in a fixed domain, i.e. with $\mathbf{g}=\mathbf{0}$. This condition will be relaxed in the next sections, when we will couple the fluid equations (1) with a model for the vessel wall in a fluid-structure interaction (FSI) model, and further on when we will couple the 3D FSI model with the 1D one.

We consider the case of Dirichlet boundary conditions (3) on $\Gamma_{i n}$ and Neumann boundary conditions (4) on $\Gamma_{a}$. In order to write problem (1) in a weak form we consider the closed subspace of $\left[H^{1}(\Omega)\right]^{3}$ of divergence free functions, which we denote by $H^{\text {div }}(\Omega)$, and we introduce the following divergence free space, for all $t \in I$ :

$$
V=\left\{\mathbf{v} \in\left[H_{\Gamma_{i n} \cup \Gamma_{w}}^{1}(\Omega)\right]^{3}: \operatorname{div} \mathbf{v}=0 \text { in } \Omega\right\}
$$

where $H_{\gamma}^{1}(\Omega)$ is the subspace of $H^{1}(\Omega)$ of functions vanishing in a measurable part $\gamma$ of the domain boundary $\partial \Omega$. The space $V$ is a closed subspace of $\left[H^{1}(\Omega)\right]^{3}$ and thus a Hilbert space for the inner product of $\left[H^{1}(\Omega)\right]^{3}$, hereafter denoted by $(\cdot, \cdot)_{H^{1}(\Omega)}$. The corresponding norm is denoted by $\|\cdot\|_{H^{1}(\Omega)}$, while $(\cdot, \cdot)_{L^{2}(\Omega)}$ and $\|\cdot\|_{L^{2}(\Omega)}$ indicate the $L^{2}(\Omega)$ inner product and norm, respectively. Sometimes, for the sake of space, we will adopt the short hand notation $(\cdot, \cdot)$ and $\|\cdot\|$ for the $L^{2}(\Omega)$ inner product and norm. To obtain the weak formulation of the Dirichlet problem, with $\mathbf{g}=\mathbf{0}$, we proceed as usual by multiplying the momentum equation of $(1)$ by $\mathbf{v} \in V$ and integrating by parts over $\Omega$. With this we obtain, for all $\mathbf{v} \in V$, for all $t \in I$, that

$$
\rho\left(\frac{\partial \mathbf{u}}{\partial t}, \mathbf{v}\right)+\frac{\rho}{2}\left\langle|\mathbf{u}|^{2}, \mathbf{v} \cdot \mathbf{n}\right\rangle_{\partial \Omega}+\rho(\operatorname{curl} \mathbf{u} \times \mathbf{u}, \mathbf{v})+\langle p, \mathbf{v} \cdot \mathbf{n}\rangle_{\partial \Omega}-2 \nu\langle\mathbf{D}(\mathbf{u}) \cdot \mathbf{n}, \mathbf{v}\rangle_{\partial \Omega}+2 \nu(\mathbf{D}(\mathbf{u}), \nabla \mathbf{v})=\mathbf{0}
$$

where $\langle\cdot, \cdot\rangle_{\gamma}$ stands for the duality pairing between $\left[H^{-1 / 2}(\gamma)\right]^{3}$ and $\left[H^{1 / 2}(\gamma)\right]^{3}$, for a regular open subset $\gamma$ of $\partial \Omega$. Taking into account the boundary conditions verified by the test functions in $V$, as well as the Neumann boundary condition on $\Gamma_{a}$, we obtain, for all $\mathbf{v} \in V$, that

$$
\rho\left(\frac{\partial \mathbf{u}}{\partial t}, \mathbf{v}\right)+2 \nu(\mathbf{D}(\mathbf{u}), \nabla \mathbf{v})+\rho(\operatorname{curl} \mathbf{u} \times \mathbf{u}, \mathbf{v})=\langle\mathbf{q}, \mathbf{v}\rangle_{\Gamma_{a}}
$$

We define, for all $t \in I$, the following bilinear and trilinear forms: for all $\mathbf{u}, \mathbf{v}, \mathbf{w} \in\left[H^{1}(\Omega)\right]^{3}$,

$$
a(\mathbf{u}, \mathbf{v}):=2 \nu(\mathbf{D}(\mathbf{u}), \nabla \mathbf{v}) \quad \text { and } \quad b(\mathbf{u}, \mathbf{v}, \mathbf{w}):=\rho(\operatorname{curl} \mathbf{u} \times \mathbf{v}, \mathbf{w}) .
$$


It is worth mentioning that both forms $a$ and $b$ are well defined in $\left[H^{1}(\Omega)\right]^{3}$, for all $t \in I$. In particular, they are continuous:

$$
\begin{aligned}
|a(\mathbf{u}, \mathbf{v})| & \leqslant 2 \nu\|\mathbf{D}(\mathbf{u})\|\|\nabla \mathbf{v}\| \leqslant 2 \nu\|\nabla \mathbf{u}\|\|\mathbf{v}\|_{H^{1}(\Omega)} \leqslant 2 \nu\|\mathbf{u}\|_{H^{1}(\Omega)}\|\mathbf{v}\|_{H^{1}(\Omega)} \\
|b(\mathbf{u}, \mathbf{v}, \mathbf{w})| & \leqslant \rho\left|\int_{\Omega}((\mathbf{v} \cdot \nabla) \mathbf{u}) \cdot \mathbf{w} \mathrm{d} \omega\right|+\rho\left|\int_{\Omega}((\nabla \mathbf{u}) \mathbf{v}) \cdot \mathbf{w} \mathrm{d} \omega\right| \leqslant 2 \rho C^{2}\|\mathbf{u}\|_{H^{1}(\Omega)}\|\mathbf{v}\|_{H^{1}(\Omega)}\|\mathbf{w}\|_{H^{1}(\Omega)}
\end{aligned}
$$

where $C$ is a positive constant, from the continuous embedding of $H^{1}(\Omega)$ in $L^{4}(\Omega)$.

We now define the following linear operator on $\left[H^{1}(\Omega)\right]^{3}$, for all $t \in I$ :

$$
L(\mathbf{v})=\langle\mathbf{q}, \mathbf{v}\rangle_{\Gamma_{a}} .
$$

Given the assumptions made on $\mathbf{q}, L$ is well defined and continuous with respect to the dual norm

$$
\|L\|_{*}=\sup _{\mathbf{v} \in V} \frac{L(\mathbf{v})}{\|\mathbf{v}\|_{H^{1}(\Omega)}} .
$$

The weak formulation of the Dirichlet problem, with $\mathbf{g}=\mathbf{0}$, reads:

Problem 2.1. Find, $\forall t \in I, \mathbf{u} \in H^{d i v}(\Omega)$ such that $\mathbf{u}(0, \cdot)=\mathbf{u}_{\mathbf{0}}(\cdot), \mathbf{u}=\mathbf{h}$ on $\Gamma_{i n}$ and

$$
\left(\frac{\partial \mathbf{u}}{\partial t}, \mathbf{v}\right)+a(\mathbf{u}, \mathbf{v})+b(\mathbf{u}, \mathbf{u}, \mathbf{v})=L(\mathbf{v}) \quad \forall \mathbf{v} \in V .
$$

For the homogeneous $(\mathbf{h}=\mathbf{0})$ steady case, i.e. $\frac{\partial \mathbf{u}}{\partial t}=0$, it is possible to derive, by a standard procedure, a well posedness result, which is given in the next Theorem 2.3. The homogeneous steady version of Problem 2.1 reads:

Problem 2.2. Find $\mathbf{u} \in V$ such that

$$
a(\mathbf{u}, \mathbf{v})+b(\mathbf{u}, \mathbf{u}, \mathbf{v})=L(\mathbf{v}) \quad \forall \mathbf{v} \in V
$$

and we have the following

Proposition 2.3. Problem 2.2 has at least one solution. Furthermore, there exists a positive constant $\delta>0$ such that if

then Problem 2.2 has a unique solution.

$$
\nu^{2}>\delta\|L\|_{*},
$$

Proof. This result can be derived from the existence and unicity results given in $[1,7]$, for the steady NavierStokes equations, applied to our problem. In those works the authors provide an existence result through a Faedo Galerkin's method for a variational problem with a trilinear form as in Problem 2.2, as long as the bilinear form $a$ is $V$-elliptic, i.e. $\exists \alpha>0$ such that $a(\mathbf{v}, \mathbf{v})>\alpha\|\mathbf{v}\|_{H^{1}(\Omega)}^{2} \forall \mathbf{v} \in V$ (see [7], Thm. 2.1).

Thus, to show the existence of solutions of Problem 2.2, we have to prove that our bilinear form $a$ is $V$-elliptic. We demonstrate it by means of the Korn (see [6]) and Poincaré (see [22]) inequalities:

$$
\forall \mathbf{v} \in\left[H_{\gamma}^{1}(\Omega)\right]^{3}, \quad\|\nabla \mathbf{v}\|^{2} \leqslant C_{K}(\Omega)(D(\mathbf{v}), D(\mathbf{v})) \quad \text { and } \quad\|\mathbf{v}\|_{H^{1}(\Omega)}^{2} \leqslant C_{P}(\Omega)(\nabla \mathbf{v}, \nabla \mathbf{v}),
$$

respectively. These inequalities can be applied to the test functions in $V$, since they vanish in a measurable part of the boundary $\partial \Omega$, recalling the assumptions made on $\Omega$. Whence, we have

$$
a(\mathbf{v}, \mathbf{v})=2 \nu(\mathbf{D}(\mathbf{v}), \nabla \mathbf{v})=2 \nu(\mathbf{D}(\mathbf{v}), \mathbf{D}(\mathbf{v})) \geqslant 2 \nu C_{K}^{-1}(\Omega)\|\nabla \mathbf{v}\|_{L^{2}(\Omega)}^{2} \geqslant C(\Omega)\|\mathbf{v}\|_{H^{1}(\Omega)}^{2} \quad \forall \mathbf{v} \in V
$$


where $C(\Omega)=2 \nu C_{K}^{-1}(\Omega)\left(C_{P}(\Omega)+1\right)^{-1}$, being $C_{K}(\Omega)$ and $C_{P}(\Omega)$ the Korn and Poincaré inequality constants, respectively.

The uniqueness, for data subject to condition (6), can be proved using the same arguments as in [7], Theorem 2.3.

It is possible to derive a similar existence and unicity result for the non homogeneous version $(\mathbf{h} \neq \mathbf{0})$ of the steady Problem 2.2 through a lifting operator procedure and a fixed point argument. In this case, further assumptions on the problem data have to be made. Precisely, the lower bound on $\nu^{2}$ in the hypothesis of the theorem depends also on the norm of the lift of $\mathbf{h}$, as well as on the coerciveness and continuity constants. We refer to [7],Theorem 2.4 for the statement of the problem and the demonstration of the result. The application to our case is straight forward.

\section{The Fluid-STRUCTURE INTERACTION PROBLEM}

To have a fluid-structure interaction description of the problem, we couple equations (1) with a model for the vessel wall. Such model provides, at any time $t \in I$, the displacement $\boldsymbol{\eta}$ with respect to the reference configuration $\Gamma_{w}^{0}$, of each point of $\Gamma_{w}$, that now depends on time $\Gamma_{w}=\Gamma_{w}(t):=\Gamma_{w}^{t}$, i.e. $\Gamma_{w}^{t}=\Gamma_{w}^{0}+\boldsymbol{\eta}$. The matching conditions between the fluid and the solid on $\Gamma_{w}^{t}$ are given by

$$
\left\{\begin{aligned}
\mathbf{u} & =\dot{\boldsymbol{\eta}} & & \text { for } t \in I \\
-\left(\boldsymbol{\sigma}(\mathbf{u}, p)+p_{\text {ext }} \mathbf{I}\right) \cdot \mathbf{n} & =\mathbf{\Phi} & & \text { for } t \in I
\end{aligned}\right.
$$

where $p_{\text {ext }}$ is a given external pressure which is considered zero from now on for the sake of simplicity and with no loss of generality, $\boldsymbol{\sigma}(\mathbf{u}, p)=-p \mathbf{I}+2 \nu \mathbf{D}(\mathbf{u})$ is the Cauchy stress tensor of the fluid, $\boldsymbol{\Phi}$ is the stress exerted by the structure on the fluid and $\mathbf{n}$ is the outward unit vector to $\Gamma_{w}^{t}$. The first of $(7)$ is the no-slip condition that guarantees the total adherence of the fluid to the structure. The second of (7) establishes the continuity of the stresses.

Depending on the type of differential operator describing the wall dynamics, one needs to provide different types of initial and boundary conditions. If the structure model is 2D (membrane model), the fluid stress on the structure is imposed through a forcing term, while if the structure model is 3D the fluid stress on the wall is imposed through a Neumann boundary condition on $\Gamma_{w}^{t}$. In both cases, the fluid stress is computed on the current configuration (Eulerian formulation), since this is the most convenient formulation for the fluid equations. However, it is common practice to write the structure equation on a reference configuration $\Gamma_{w}^{0}$ (Lagrangian formulation), which is usually taken to be that at the initial time. Thus, it is useful to rewrite the continuity of the stresses (the second of $(7)$ ) on $\Gamma_{w}^{0}$. By means of the Piola transform (see [6]), we have

$$
-\left(\operatorname{det} \nabla_{\mathbf{0}} \boldsymbol{\eta}\right)\left(\boldsymbol{\sigma}(\mathbf{u}, p)+p_{\text {ext }} \mathbf{I}\right)\left(\nabla_{\mathbf{0}}^{-T} \boldsymbol{\eta}\right) \cdot \mathbf{n}_{0}=\widehat{\boldsymbol{\Phi}} \quad \text { for } t \in I, \text { on } \Gamma_{w}^{0},
$$

where $\widehat{\boldsymbol{\Phi}}$ is the structure force per unit reference surface area on $\Gamma_{w}^{0}, \nabla_{\mathbf{0}}$ indicates the gradient with respect to the Lagrangian coordinates, and $\mathbf{n}_{0}$ is the outward unit vector to $\Gamma_{w}^{0}$.

By coupling the fluid equations with a structural wall model, the domain now changes in time $(\Omega=\Omega(t):=$ $\Omega^{t}$ ) with the solution of the structure problem $\boldsymbol{\eta}$. Thus, the domain regularity depends on the regularity of the vessel wall model. Results on the regularity of the solution of a FSI problem, which would ensure the regularity of the fluid-structure interface, are still missing, as we will discuss later on. We then need to do some hypothesis on the regularity of the domain a priori. Precisely, we assume that, at any time $t \in \bar{I}$ the domain $\Omega^{t} \subset \mathbb{R}^{3}$ is open and connected and its boundary $\partial \Omega^{t}:=\Gamma_{w}^{t} \cup \Gamma_{i n}^{t} \cup \Gamma_{a}^{t}$ is locally Lipschitz (i.e., $\partial \Omega^{t} \in \mathcal{C}^{1,1}$ ). Furthermore, we assume that the domain $\Omega^{t}$ verifies the cone property, that will be needed to apply Korn's inequality (see [23]).

Moreover, in order to derive the stability results in the sequel, we need to apply a trace and Korn (see [6,23]) inequalities. Since the constants on those inequalities depend on the domain, which in turn depends on the 


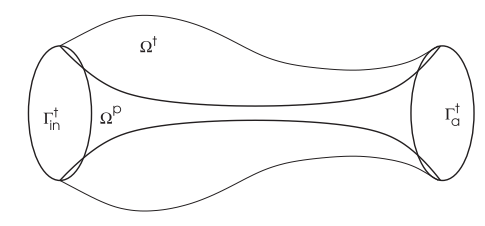

Figure 2. Domain $\Omega^{p}$, subset of $\Omega^{t}$, for all $t \in I$.

solution $\boldsymbol{\eta}$ itself, we need to assume that those constants, for all $t \in \bar{I}$, are uniformly bounded from above. Precisely, we assume the following.

Hypothesis 3.1. There exist positive constants $C_{i n}$ and $C_{a}$ such that, for all $\mathbf{v} \in\left[H^{1}\left(\Omega^{t}\right)\right]^{3}$

$$
\|\mathbf{v}\|_{L^{2}\left(\Gamma_{i n}^{t}\right)} \leqslant C_{i n}\left(\|\mathbf{v}\|_{L^{2}\left(\Omega^{t}\right)}^{2}+\|\mathbf{D}(\mathbf{v})\|_{L^{2}\left(\Omega^{t}\right)}^{2}\right)^{\frac{1}{2}}, \quad \text { and } \quad\|\mathbf{v}\|_{L^{2}\left(\Gamma_{a}^{t}\right)} \leqslant C_{a}\left(\|\mathbf{v}\|_{L^{2}\left(\Omega^{t}\right)}^{2}+\|\mathbf{D}(\mathbf{v})\|_{L^{2}\left(\Omega^{t}\right)}^{2}\right)^{\frac{1}{2}} .
$$

Remark 3.2. For all $\mathbf{v} \in\left[H^{1}\left(\Omega^{t}\right)\right]^{3}$ vanishing in a measurable part of the boundary $\partial \Omega^{t}$, a stronger Korn inequality (see [6], Thms. 6.3 and 6.4) can be applied, and Hypothesis 3.1 becomes: there exist positive constants $\widetilde{C}_{i n}$ and $\widetilde{C}_{a}$ such that

$$
\|\mathbf{v}\|_{L^{2}\left(\Gamma_{i n}^{t}\right)} \leqslant \widetilde{C}_{i n}\|\mathbf{D}(\mathbf{v})\|_{L^{2}\left(\Omega^{t}\right)} \quad \text { and } \quad\|\mathbf{v}\|_{L^{2}\left(\Gamma_{a}^{t}\right)} \leqslant \widetilde{C}_{a}\|\mathbf{D}(\mathbf{u})\|_{L^{2}\left(\Omega^{t}\right)} .
$$

Proposition 3.3. If sections $\Gamma_{\text {in }}^{t}$ and $\Gamma_{a}^{t}$ do not change in time, and if the domain $\Omega^{t}$ is regular enough, such that there exists a Lipschitz subdomain $\Omega^{p}$ of $\Omega^{t}$, verifying the cone property, which is included in every $\Omega^{t}$, for all $t \in \bar{I}$, and whose boundary contains $\Gamma_{\text {in }}^{t}$ and $\Gamma_{a}^{t}$ (see Fig. 2), then Hypothesis 3.1 is verified.

Proof. Due to the characteristics of $\Omega^{p}$ and its independence on the time $t$, the Korn inequality is valid in $\Omega^{p}$, at any time $t \in I$, for all $\mathbf{v} \in\left[H^{1}\left(\Omega^{p}\right)\right]^{3}$ (see [6,23]). Moreover, since $\Gamma_{a}^{t} \cup \Gamma_{i n}^{t} \subset \partial \Omega^{p}$, for all $t \in \bar{I}$, we have a trace inequality on $\Omega^{p}$. Whence the following inequalities hold, for all $\mathbf{v} \in\left[H^{1}\left(\Omega^{t}\right)\right]^{3}$ :

$$
\begin{aligned}
\|\mathbf{v}\|_{L^{2}\left(\Gamma_{i n}^{t}\right)} & \left.\leqslant \gamma_{i n}\|\mathbf{v}\|_{H^{1}\left(\Omega^{p}\right)} \leqslant C_{i n}\left(\|\mathbf{v}\|_{L^{2}\left(\Omega^{p}\right)}^{2}+\|\mathbf{D}(\mathbf{v})\|_{L^{2}\left(\Omega^{p}\right)}^{2}\right)^{\frac{1}{2}} \leqslant C_{i n}\left(\|\mathbf{v}\|_{L^{2}\left(\Omega^{t}\right)}^{2}+\|\mathbf{D}(\mathbf{v})\|_{L^{2}\left(\Omega^{t}\right)}^{2}\right)\right)^{\frac{1}{2}} \\
\|\mathbf{v}\|_{L^{2}\left(\Gamma_{a}^{t}\right)} & \leqslant \gamma_{a}\|\mathbf{v}\|_{H^{1}\left(\Omega^{p}\right)} \leqslant C_{a}\left(\|\mathbf{v}\|_{L^{2}\left(\Omega^{p}\right)}^{2}+\|\mathbf{D}(\mathbf{v})\|_{L^{2}\left(\Omega^{p}\right)}^{2}\right)^{\frac{1}{2}} \leqslant C_{a}\left(\|\mathbf{v}\|_{L^{2}\left(\Omega^{t}\right)}^{2}+\|\mathbf{D}(\mathbf{v})\|_{L^{2}\left(\Omega^{t}\right)}^{2}\right)^{\frac{1}{2}}
\end{aligned}
$$

where $C_{i n}=C_{i n}\left(\Omega^{p}\right)=\gamma_{i n} C_{K}$ and $C_{a}=C_{a}\left(\Omega^{p}\right)=\gamma_{a} C_{K}$ are independent of time, with $\gamma_{i n}=\gamma_{i n}\left(\Omega^{p}\right)$ and $\gamma_{a}=\gamma_{a}\left(\Omega^{p}\right)$ the trace inequality constants and $C_{K}=C_{K}\left(\Omega^{p}\right)$ the Korn inequality constant.

Observe that, if the hypothesis of Proposition 3.3 are verified and $\mathbf{v}$ vanishes in a measurable portion of the boundary $\partial \Omega^{p}$, then inequalities of Remark 3.2 hold true, with $\widetilde{C}_{i n}=\widetilde{C}_{i n}\left(\Omega^{p}\right)$ and $\widetilde{C}_{a}=\widetilde{C}_{a}\left(\Omega^{p}\right)$ independent of time.

In this work we will analyze different models to represent the movements of the vessel wall, starting with a simple algebraic one and concluding with a 3D non linear elasticity model. 


\subsection{Simple 2D structure models}

We will start by considering simple $2 \mathrm{D}$ structure models accounting only for radial movements, i.e., $\boldsymbol{\eta}=\eta_{r} \mathbf{e}_{r}$ where $\mathbf{e}_{r}$ is the radial coordinate unit vector and $\eta_{r}$ the radial component of $\boldsymbol{\eta}$. In this case, the reference configuration $\Gamma_{w}^{0}$ is assumed to have a cylindrical shape, so that the meaning of radial movement is well understood:

$$
\Gamma_{w}^{0}=\left\{(r, \theta, z): r=R_{0}, 0 \leqslant z \leqslant a, 0 \leqslant \theta<2 \pi\right\},
$$

where $R_{0}$ is the arterial reference radius at rest and might depend on the axial coordinate $z$.

Since only radial movements are considered, we take only the radial component of the structural wall stresses $\widehat{\Phi}_{r}=\widehat{\mathbf{\Phi}} \cdot \mathbf{e}_{r}$. Hence, the structural model may be written in the form

$$
S\left(\eta_{r}\right)=\widehat{\Phi}_{r},
$$

where $S$ is an operator, either differential or algebraic, that identifies the specific model at hand.

We first consider a simple pressure-area algebraic relationship, which is the simplest model derived from the equations of elasticity, assuming a cylindrical geometry and membrane deformations. The thickness of the wall is assumed to be small and vessel inertia, elastic bending as well as shear stress terms are neglected. The model is then given by

$$
\mathcal{S}\left(\eta_{r}\right):=\frac{E h}{R_{0}^{2}\left(1-\xi^{2}\right)} \eta_{r}=b \eta_{r}
$$

being $h$ the wall thickness, $E$ the Young modulus and $\xi$ the Poisson ratio.

Defining the energy of the coupled problem (1), (2), (7) and (11) as

$$
\mathscr{E}_{3 D-A L}(t):=\frac{\rho}{2}\|\mathbf{u}\|_{L^{2}\left(\Omega^{t}\right)}^{2}+\frac{b}{2}\left\|\eta_{r}\right\|_{L^{2}\left(\Gamma_{w}^{0}\right)}^{2}
$$

the following result holds.

Theorem 3.4. The coupled fluid-structure problem (1), (2), (7) and (11), satisfies the following equality:

$$
\frac{\mathrm{d}}{\mathrm{d} t}\left(\mathscr{E}_{3 D-A L}(t)\right)+2 \nu\|\mathbf{D}(\mathbf{u})\|_{L^{2}\left(\Omega^{t}\right)}^{2}=\int_{\Gamma_{i n}^{t} \cup \Gamma_{a}^{t}}\left(\boldsymbol{\sigma}^{t o t}(\mathbf{u}, p) \cdot \mathbf{n}\right) \cdot \mathbf{u} \mathrm{d} \gamma,
$$

by which we obtain:

$$
\mathscr{E}_{3 D-A L}(T)+2 \nu \int_{0}^{T}\|\mathbf{D}(\mathbf{u})\|_{L^{2}\left(\Omega^{t}\right)}^{2} \mathrm{~d} t=\mathscr{E}_{3 D-A L}(0)+\int_{0}^{T} \int_{\Gamma_{i n}^{t} \cup \Gamma_{a}^{t}}\left(\boldsymbol{\sigma}^{t o t}(\mathbf{u}, p) \cdot \mathbf{n}\right) \cdot \mathbf{u} \mathrm{d} \gamma \mathrm{d} t
$$

where $\mathscr{E}_{3 D-A L}(0)$ is a constant depending only on the initial datum $\mathbf{u}_{0}$.

Proof. We first multiply the structure equation (11) by $\frac{\partial \eta_{r}}{\partial t}$ and integrate on the reference cylindrical surface $\Gamma_{w}^{0}$, obtaining

$$
b \int_{\Gamma_{w}^{0}} \eta_{r} \frac{\partial \eta_{r}}{\partial t} \mathrm{~d} \gamma=\frac{b}{2} \int_{\Gamma_{w}^{0}} \frac{\partial}{\partial t}\left(\eta_{r}\right)^{2} \mathrm{~d} \gamma=\frac{b}{2} \frac{\mathrm{d}}{\mathrm{d} t}\left\|\eta_{r}\right\|_{L^{2}\left(\Gamma_{w}^{0}\right)}^{2}
$$

Regarding the forcing term of the structure, thanks to the continuity of the stresses on $\Gamma_{w}^{0}$ given in (8), and the continuity of the velocities on $\Gamma_{w}^{t}$ (the first of (7)), we have:

$$
\int_{\Gamma_{w}^{0}} \widehat{\Phi}_{r} \frac{\partial \eta_{r}}{\partial t} \mathrm{~d} \gamma=\int_{\Gamma_{w}^{0}}\left(-\left(\operatorname{det} \nabla_{\mathbf{0}} \boldsymbol{\eta}\right)(\boldsymbol{\sigma}(\mathbf{u}, p))\left(\nabla_{\mathbf{0}}^{-T} \boldsymbol{\eta}\right) \cdot \mathbf{n}_{0}\right) \cdot \mathbf{e}_{r} \frac{\partial \eta_{r}}{\partial t} \mathrm{~d} \gamma=-\int_{\Gamma_{w}^{t}}(\boldsymbol{\sigma}(\mathbf{u}, p) \cdot \mathbf{n}) \cdot \mathbf{u d} \gamma
$$


Whence we obtain:

$$
\frac{b}{2} \frac{\mathrm{d}}{\mathrm{d} t}\left\|\eta_{r}\right\|_{L^{2}\left(\Gamma_{w}^{0}\right)}^{2}=-\int_{\Gamma_{w}^{t}}(\boldsymbol{\sigma}(\mathbf{u}, p) \cdot \mathbf{n}) \cdot \mathbf{u} \mathrm{d} \gamma
$$

Similarly for the fluid equations, we multiply the momentum equation by $\mathbf{u}$ and integrate over $\Omega^{t}$. We first observe that, due to the no-slip condition (the first of (7)), the boundary $\Gamma_{w}^{t}$ of the fluid domain $\Omega^{t}$ is moving with velocity $\mathbf{u}$ and thus, from the Reynolds transport theorem, we may write

$$
\int_{\Omega^{t}} \rho \frac{1}{2} \frac{\partial}{\partial t}|\mathbf{u}|^{2} \mathrm{~d} \omega=\frac{\rho}{2} \frac{\mathrm{d}}{\mathrm{d} t} \int_{\Omega^{t}}|\mathbf{u}|^{2} \mathrm{~d} \gamma-\frac{\rho}{2} \int_{\Gamma_{w}^{t}}|\mathbf{u}|^{2} \mathbf{u} \cdot \mathbf{n} \mathrm{d} \gamma=\frac{\rho}{2} \frac{\mathrm{d}}{\mathrm{d} t}\|\mathbf{u}\|_{L^{2}\left(\Omega^{t}\right)}^{2}-\frac{\rho}{2} \int_{\Gamma_{w}^{t}}|\mathbf{u}|^{2} \mathbf{u} \cdot \mathbf{n} \mathrm{d} \gamma .
$$

On the other hand, noticing that

$$
\int_{\Omega^{t}}(\operatorname{curl} \mathbf{u} \times \mathbf{u}) \cdot \mathbf{u} \mathrm{d} \omega=\int_{\Omega^{t}}\left((\mathbf{u} \cdot \nabla) \mathbf{u}-\frac{1}{2} \nabla|\mathbf{u}|^{2} \mathbf{u}\right) \mathbf{u} \mathrm{d} \omega=\int_{\Omega^{t}}\left(\frac{1}{2} \mathbf{u} \cdot \nabla|\mathbf{u}|^{2}-\frac{1}{2} \mathbf{u} \cdot \nabla|\mathbf{u}|^{2}\right) \mathrm{d} \omega=0,
$$

and that

and thanks to the incompressibility condition $\operatorname{div} \mathbf{u}=0$, we have:

$$
\int_{\Omega^{t}} \mathbf{D}(\mathbf{u}): \nabla \mathbf{u} \mathrm{d} \omega=\int_{\Omega^{t}} \mathbf{D}(\mathbf{u}): \mathbf{D}(\mathbf{u}) \mathrm{d} \omega
$$

$$
\frac{\rho}{2} \frac{\mathrm{d}}{\mathrm{d} t}\|\mathbf{u}\|_{L^{2}\left(\Omega^{t}\right)}^{2}+2 \nu\|\mathbf{D}(\mathbf{u})\|_{L^{2}\left(\Omega^{t}\right)}^{2}=\int_{\Gamma_{i n}^{t} \cup \Gamma_{a}^{t}}\left(\boldsymbol{\sigma}^{t o t}(\mathbf{u}, p) \cdot \mathbf{n}\right) \cdot \mathbf{u} \mathrm{d} \gamma+\int_{\Gamma_{w}^{t}}(\boldsymbol{\sigma}(\mathbf{u}, p) \cdot \mathbf{n}) \cdot \mathbf{u} \mathrm{d} \gamma
$$

Summing up (14) and (15) we derive (12), and then (13) by integration between 0 and $T$.

If homogeneous boundary conditions, either Dirichlet or Neumann, are prescribed in the artificial sections, the boundary terms on the estimates (12) and (13) vanish, leading to the following energy decay property.

Corollary 3.5 (energy decay property). The coupled fluid-structure problem (1), (2), (7) and (11), with homogeneous boundary conditions on $\Gamma_{\text {in }}^{t}$ and $\Gamma_{a}^{t}$, satisfies the following energy equality:

$$
\frac{\mathrm{d}}{\mathrm{d} t}\left(\mathscr{E}_{3 D-A L}(t)\right)+2 \nu\|\mathbf{D}(\mathbf{u})\|_{L^{2}\left(\Omega^{t}\right)}^{2}=0
$$

by which we obtain the following energy decay property:

$$
\mathscr{E}_{3 D-A L}(T)+2 \nu \int_{0}^{T}\|\mathbf{D}(\mathbf{u})\|_{L^{2}\left(\Omega^{t}\right)}^{2} \mathrm{~d} t=\mathscr{E}_{3 D-A L}(0),
$$

where $\mathscr{E}_{3 D-A L}(0)$ is a constant depending only on the initial datum $\mathbf{u}_{0}$.

If Neumann boundary conditions are considered on the artificial sections:

$$
\boldsymbol{\sigma}^{t o t}(\mathbf{u}, p) \cdot \mathbf{n}=\mathbf{r} \text { on } \Gamma_{i n}^{t}, \quad \text { and } \quad \boldsymbol{\sigma}^{t o t}(\mathbf{u}, p) \cdot \mathbf{n}=\mathbf{q} \text { on } \Gamma_{a}^{t},
$$

an energy estimate for the fluid-structure problem can be derived by means of the Gronwall's inequality.

Theorem 3.6 (Neumann problem). Under the Hypothesis 3.1, the coupled fluid-structure problem (1), (2), (18), (7) and (11), satisfies the following a priori energy estimate, for all $t \in I$

$$
\mathscr{E}_{3 D-A L}(t)+\nu \int_{0}^{t}\|\mathbf{D}(\mathbf{u})\|_{L^{2}\left(\Omega^{t}\right)}^{2} \mathrm{~d} t \leqslant\left(\mathscr{E}_{3 D-A L}(0)+\widetilde{C} \int_{0}^{t}\|\mathbf{r}\|_{L^{2}\left(\Gamma_{i n}^{t}\right)}^{2} \mathrm{~d} t+C \int_{0}^{t}\|\mathbf{q}\|_{L^{2}\left(\Gamma_{a}^{t}\right)}^{2} \mathrm{~d} t\right) \mathrm{e}^{\frac{2 \nu}{\rho} t},
$$

where $\widetilde{C}$ and $C$ are positive constants, and $\mathscr{E}_{3 D-A L}(0)$ is a constant depending only on the initial datum $\mathbf{u}_{0}$. 
Proof. Applying the inequalities of Hypothesis 3.1, and twice the Young inequality $a b \leqslant \frac{1}{2 \beta} a^{2}+\frac{\beta}{2} b^{2}$, with $\beta=\frac{\nu}{C_{i n}}$ and $\beta=\frac{\nu}{C_{a}}$, being $C_{i n}$ and $C_{a}$ the constants in inequalities of Hypothesis 3.1, we have:

$$
\begin{aligned}
\int_{\Gamma_{i n}^{t} \cup \Gamma_{a}^{t}}\left(\boldsymbol{\sigma}^{t o t}(\mathbf{u}, p) \cdot \mathbf{n}\right) \cdot \mathbf{u} \mathrm{d} \gamma & \leqslant\|\mathbf{u}\|_{L^{2}\left(\Gamma_{i n}^{t}\right)}\|\mathbf{r}\|_{L^{2}\left(\Gamma_{i n}^{t}\right)}+\|\mathbf{u}\|_{L^{2}\left(\Gamma_{a}^{t}\right)}\|\mathbf{q}\|_{L^{2}\left(\Gamma_{a}^{t}\right)} \\
& \leqslant \frac{\nu}{2 C_{i n}}\|\mathbf{u}\|_{L^{2}\left(\Gamma_{i n}^{t}\right)}^{2}+\widetilde{C}\|\mathbf{r}\|_{L^{2}\left(\Gamma_{i n}^{t}\right)}^{2}+\frac{\nu}{2 C_{a}}\|\mathbf{u}\|_{L^{2}\left(\Gamma_{a}^{t}\right)}^{2}+C\|\mathbf{q}\|_{L^{2}\left(\Gamma_{a}^{t}\right)}^{2} \\
& \leqslant \nu\|\mathbf{u}\|_{L^{2}\left(\Omega^{t}\right)}^{2}+\nu\|\mathbf{D}(\mathbf{u})\|_{L^{2}\left(\Omega^{t}\right)}^{2}+\widetilde{C}\|\mathbf{r}\|_{L^{2}\left(\Gamma_{i n}^{t}\right)}^{2}+C\|\mathbf{q}\|_{L^{2}\left(\Gamma_{a}^{t}\right)}^{2},
\end{aligned}
$$

where $\widetilde{C}=\frac{C_{i n}}{2 \nu}$ and $C=\frac{C_{a}}{2 \nu}$.

Whence, integrating (12) between 0 and $t \leqslant T$ we obtain the following inequality

$$
\mathscr{E}_{3 D-A L}(t)+\nu \int_{0}^{t}\|\mathbf{D}(\mathbf{u})\|_{L^{2}\left(\Omega^{t}\right)}^{2} \mathrm{~d} t \leqslant \mathscr{E}_{3 D-A L}(0)+\widetilde{C} \int_{0}^{t}\|\mathbf{r}\|_{L^{2}\left(\Gamma_{i n}^{t}\right)}^{2} \mathrm{~d} t+C \int_{0}^{t}\|\mathbf{q}\|_{L^{2}\left(\Gamma_{a}^{t}\right)}^{2} \mathrm{~d} t+\nu \int_{0}^{t}\|\mathbf{u}\|_{L^{2}\left(\Omega^{t}\right)}^{2} \mathrm{~d} t
$$

We now apply a Gronwall inequality (see for instance [6]): if $g$ and $\psi$ are continuous functions in $I$ and $\psi(t) \leqslant g(t)+c \int_{0}^{t} \psi(s) \mathrm{d} s$, then $\psi(t) \leqslant g(t) \mathrm{e}^{c t}$. The desired inequality is obtained by identifying

$$
\psi(t)=\mathscr{E}_{3 D-A L}(t)+\nu \int_{0}^{t}\|\mathbf{D}(\mathbf{u})\|_{L^{2}\left(\Omega^{t}\right)}^{2} \mathrm{~d} t,
$$

and noticing that

$$
\nu \int_{0}^{t}\|\mathbf{u}\|_{L^{2}\left(\Omega^{t}\right)}^{2} \mathrm{~d} t \leqslant \frac{2 \nu}{\rho} \int_{0}^{t} \psi(s) \mathrm{d} s
$$

Remark 3.7. The need for the application of a Gronwall inequality for the Neumann problem is due to the fact that imposing Neumann boundary conditions both on $\Gamma_{i n}^{t}$ and $\Gamma_{a}^{t}$ does not prevent the existence of rigid motions. In fact, the solution of the problem is well defined due to the fulfillment of the initial condition, yet, asymptotically, the solution is defined only up to a rigid motion and we might loose control on the energy of the system in terms of the forcing terms. If we assume that the two sections $\Gamma_{i n}^{t}$ and $\Gamma_{a}^{t}$ are fixed, this implies, in particular, that $\mathbf{u}=\mathbf{0}$ on $\partial \Gamma_{i n}^{t}$ and $\partial \Gamma_{a}^{t}$, which would be enough to prevent rigid body motions. However, the trace of a function $\mathbf{u} \in\left[H^{1}\left(\Omega^{t}\right)\right]^{3}$ on a one-dimensional manifold is not well defined, and the use of the more stringent Korn inequality (9) (see Rem. 3.2) is questionable. This issue is still open.

Remark 3.8. The stability result is not derived for the non homogeneous Dirichlet problem since in that case a lifting procedure has to be carried out and the Hopf Lemma (see [22], Lem. 2.3, Chap. IV) applied. The extension of either of them to the moving domain case and for a trilinear form as the one used in this work is not immediate.

If we include a second time derivative on the pressure-area algebraic model (11) accounting for the vessel wall inertia, we obtain the independent rings model [33], where

$$
S\left(\eta_{r}\right):=\tilde{\rho}_{w} \frac{\partial^{2} \eta_{r}}{\partial t^{2}}+b \eta_{r}
$$

being $\tilde{\rho}_{w}=\rho_{w} h$, with $\rho_{w}$ the density of the wall material. This model needs suitable initial conditions:

$$
\eta_{r}=\eta_{0} \quad \text { and } \quad \dot{\eta}_{r}=\dot{\eta}_{0}, \quad \text { for } \quad t=0, \quad \text { on } \quad \Gamma_{w}^{0},
$$


where the initial velocity $\dot{\eta}_{0}$ has to be compatible with the fluid initial condition: $\dot{\eta}_{0} \mathbf{e}_{r}=\mathbf{u}_{0}$.

Similar results to Theorems $3.4,3.5$ and 3.6 can be obtained in this case by substituting $\mathscr{E}_{3 D-A L}(t)$ with $\mathscr{E}_{3 D-I R}(t)$ defined as

$$
\mathscr{E}_{3 D-I R}(t):=\frac{\rho}{2}\|\mathbf{u}\|_{L^{2}\left(\Omega^{t}\right)}^{2}+\frac{\tilde{\rho}_{w}}{2}\left\|\frac{\partial \eta_{r}}{\partial t}\right\|_{L^{2}\left(\Gamma_{w}^{0}\right)}^{2}+\frac{b}{2}\left\|\eta_{r}\right\|_{L^{2}\left(\Gamma_{w}^{0}\right)}^{2}
$$

and noticing that

$$
\tilde{\rho}_{w} \int_{\Gamma_{w}^{0}} \frac{\partial^{2} \eta_{r}}{\partial t^{2}} \frac{\partial \eta_{r}}{\partial t} \mathrm{~d} \gamma=\frac{\tilde{\rho}_{w}}{2} \frac{\mathrm{d}}{\mathrm{d} t}\left\|\frac{\partial \eta_{r}}{\partial t}\right\|_{\Gamma_{w}^{0}}^{2} .
$$

Continuing to increase complexity, we add to the independent rings model a shear stress term, obtaining a generalized string model, for which

$$
S\left(\eta_{r}\right):=\tilde{\rho}_{w} \frac{\partial^{2} \eta_{r}}{\partial t^{2}}-a \frac{\partial^{2} \eta_{r}}{\partial z^{2}}+b \eta_{r}
$$

where $a=K G h$, with $K$ the Timoshenko shear correction factor and $G$ the shear modulus. In this model, longitudinal movements are still neglected, but shear forces are modeled. The shear stress term introduces second order spatial derivatives, making it necessary to set up boundary conditions at $\partial \Gamma_{w}^{t}=\left(\Gamma_{w}^{t} \cap \Gamma_{i n}^{t}\right) \cup\left(\Gamma_{w}^{t} \cap \Gamma_{a}^{t}\right)$.

The energy of the coupled FSI problem in this case is defined by

$$
\mathscr{E}_{3 D-G S}(t):=\frac{\rho}{2}\|\mathbf{u}\|_{L^{2}\left(\Omega^{t}\right)}^{2}+\frac{\tilde{\rho}_{w}}{2}\left\|\frac{\partial \eta_{r}}{\partial t}\right\|_{L^{2}\left(\Gamma_{w}^{0}\right)}^{2}+\frac{a}{2}\left\|\frac{\partial \eta_{r}}{\partial z}\right\|_{L^{2}\left(\Gamma_{w}^{0}\right)}^{2}+\frac{b}{2}\left\|\eta_{r}\right\|_{L^{2}\left(\Gamma_{w}^{0}\right)}^{2} .
$$

Observing that

$$
-a \int_{\Gamma_{w}^{0}} \frac{\partial^{2} \eta_{r}}{\partial z^{2}} \frac{\partial \eta_{r}}{\partial t} \mathrm{~d} \gamma=\frac{a}{2} \frac{\mathrm{d}}{\mathrm{d} t}\left\|\frac{\partial \eta_{r}}{\partial z}\right\|_{L^{2}\left(\Gamma_{w}^{0}\right)}^{2}-\int_{\partial \Gamma_{w}^{0}}\left(a \frac{\partial \eta_{r}}{\partial z} \frac{\partial \eta_{r}}{\partial t}\right) \mathrm{d} \gamma
$$

similar results to those of Theorems 3.5 and 3.6 can be deduced by imposing Neumann homogeneous boundary conditions $a \frac{\partial \eta_{r}}{\partial z}=0$ on $\partial \Gamma_{w}^{0}$, for all $t \in I$.

We conclude this section by giving some remarks on the models studied here. These are very simple models for the wall movement which in principle do not guarantee sufficient regularity of the solution for the coupling with the fluid equations. Thus, not even the existence of the coupled problem can be demonstrated rigorously, or at least no proof in that direction is available so far. Indeed, from the matching conditions (7), we see that the fluid velocity at the interface boundary $\Gamma_{w}^{t}$ with the vessel is given by the time derivative of the wall displacement $\eta_{r}$. However, for the models here presented to describe the wall displacement, the time derivative of the solution $\dot{\eta}_{r}(t)$ does not, in general, belong to $H^{1 / 2}\left(\Gamma_{w}^{0}\right)$ which is the natural space for the trace of $\mathbf{u}(t)$ in $\Gamma_{w}^{t}$. For instance, for the algebraic law model the regularity of the structure displacement $\eta_{r}$ coincides with the regularity of the forcing term $\Phi_{r}$. But the forcing term $\Phi_{r}$, due to the matching conditions (7), is given by the radial component of the normal stress exerted by the fluid which, in the minimal conditions for the Navier-Stokes problem, is only a $H^{-1 / 2}$ functional.

For this reason, in order to justify our results, we have to assume a priori that the displacement $\eta_{r}$ is sufficiently regular. In particular, that $\frac{\partial \eta_{r}}{\partial t}(t) \in H^{1 / 2}\left(\Gamma_{w}^{0}\right)$, which guarantees that $\mathbf{u}(t) \in H^{1}\left(\Omega^{t}\right)$ and makes all the integrals and norms used to derive the above results well defined. Furthermore, as already mentioned, since the fluid domain $\Omega^{t}$ depends on the structure model solution, we assumed that such solution is regular enough so that $\Omega^{t}$ is sufficiently regular at all times. The issue of the regularity of the structure solution, and consequently of the regularity, existence and uniqueness of the fluid-structure interaction problem, is still an open problem. 


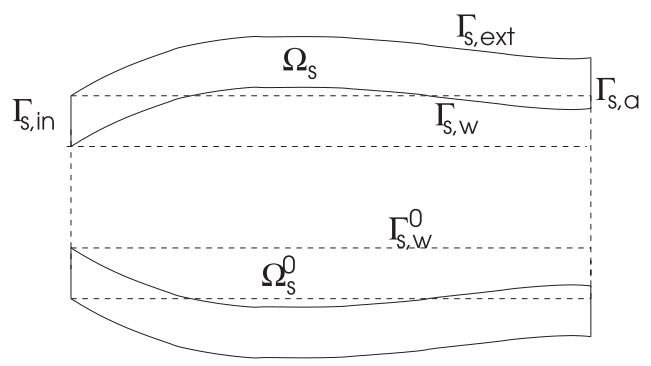

FiguRE 3. Longitudinal section of the reference $\Omega_{s}^{0}$ and current $\Omega_{s}^{t}$ structure domains.

To augment the regularity of the problem, several authors $[2,5,9,37]$ have added to the generalized strings model extra regularizing terms, proportional to $\frac{\partial^{3} \eta_{r}}{\partial z^{2} \partial t}, \frac{\partial^{4} \eta_{r}}{\partial z^{4}}$, or even $\frac{\partial^{5} \eta_{r}}{\partial z^{4} \partial t}$. These terms may guarantee the required regularity of the time derivative of the displacement, however they do not have a direct physical interpretation.

We shall see from numerical evidence that despite this fact, the simple models here presented seem to work very well in practice.

\subsection{D non-linear elastic model}

We consider now the equations of 3D non-linear elasticity to describe the wall dynamics. Unlike the previous models, here the structure domain is a $3 \mathrm{D}$ bounded subset $\Omega_{s}^{t}=\Omega_{s}(t)$ of $\mathbb{R}^{3}$, for $t \in I$, with boundary $\partial \Omega_{s}^{t}=$ $\partial \Omega_{s}(t)$. We subdivide the boundary into four disjoint parts, such that $\partial \Omega_{s}^{t}=\partial \Omega_{s}(t)=\Gamma_{s, w}^{t} \cup \Gamma_{s, e x t}^{t} \cup \Gamma_{s, i n}^{t} \cup \Gamma_{s, a}^{t}$, where $\Gamma_{s, w}^{t}$ is the part of the boundary interfacing with the fluid domain, $\Gamma_{s, e x t}^{t}$ is the part in contact with the exterior and $\Gamma_{s, i n}^{t}$ and $\Gamma_{s, a}^{t}$ denote the artificial parts of the boundary at the inflow and outflow sections, respectively (see Fig. 3). $\Omega_{s}^{0}$ is the structure reference domain, which is taken to be the wall domain at the initial time, when we assume that the wall is at rest, and $\partial \Omega_{s}^{0}=\Gamma_{s, w}^{0} \cup \Gamma_{s, e x t}^{0} \cup \Gamma_{s, i n}^{0} \cup \Gamma_{s, a}^{0}$ is the corresponding reference boundary.

The 3D equations of elasticity on $\Omega_{s}^{t}$ have to be endowed with initial and boundary conditions. We take the following initial conditions:

$$
\boldsymbol{\eta}=\boldsymbol{\eta}_{0} \quad \text { and } \quad \dot{\boldsymbol{\eta}}=\dot{\boldsymbol{\eta}}_{0}, \quad \text { for } \quad t=0, \quad \text { in } \quad \Omega_{s}^{0}
$$

The initial velocity $\dot{\boldsymbol{\eta}}_{0}$ has to be compatible with the fluid initial condition, namely

$$
\dot{\boldsymbol{\eta}}_{0}=\mathbf{u}_{0}, \quad \text { on } \quad \Gamma_{w}^{0}
$$

At the exterior boundary $\Gamma_{s, \text { ext }}^{t}$ we assume that the stress is zero, so we take a homogeneous Neumann boundary condition. At $\Gamma_{s, w}^{t}$ we have the matching conditions (7). At the inflow and outflow sections it is not clear, from physical arguments, what is the proper boundary condition to impose. As we have seen previously for the generalized strings model, these boundaries introduce a boundary term in the energy estimate of the FSI problem, which vanishes if homogeneous boundary conditions are considered. For the sake of simplicity we take a Dirichlet boundary condition at the upstream section $\Gamma_{s, i n}^{t}$, meaning that the artery is clamped at the inflow. At the downstream section $\Gamma_{s, a}^{t}$ we take a homogeneous Neumman boundary condition on the radial direction and a homogeneous Dirichlet boundary condition on the longitudinal direction, meaning that the structure can not move on the longitudinal direction but it is free to move radially. 
As it is customary in solid mechanics, we write the structure equations in Lagrangian coordinates (i.e. with respect to the reference configuration). The structure model, for $3 \mathrm{D}$ compressible elastic materials, is thus given by

$$
\left\{\begin{array}{rlll}
\rho_{w} \frac{\partial^{2} \boldsymbol{\eta}}{\partial t^{2}}-\operatorname{div}_{0}(\mathbf{P}) & =\mathbf{0} & & \text { on } \Omega_{s}^{0} \\
\mathbf{P} \cdot \mathbf{n}_{0} & =\widehat{\boldsymbol{\Phi}} & & \text { on } \Gamma_{w}^{0} \\
\mathbf{P} \cdot \mathbf{n}_{0} & =\mathbf{0} & & \text { on } \Gamma_{s, e x t}^{0} \\
\left(\mathbf{P} \cdot \mathbf{n}_{0}\right) \cdot \boldsymbol{\tau}_{a} & =0 & & \text { on } \Gamma_{s, a}^{0} \\
\boldsymbol{\eta} \cdot \mathbf{n}_{a} & =0 & & \text { on } \Gamma_{s, a}^{0} \\
\boldsymbol{\eta} & =\mathbf{0} & & \text { on } \Gamma_{s, i n}^{0}
\end{array}\right.
$$

where $\rho_{w}$ is the wall density, $\mathbf{n}_{0}$ is the outward unit vector to the reference configuration $\Omega_{s}^{0}, \boldsymbol{\tau}_{a}$ and $\mathbf{n}_{a}$ are the tangent and outward normal vectors to $\Gamma_{s, a}^{0}$, respectively; $\operatorname{div}_{0}$ stands for the divergence operator with respect to the Lagrangian coordinates and $\mathbf{P}=\mathbf{P}(\boldsymbol{\eta})=\mathbf{F S}$ is the first Piola-Kirchhoff tensor, with $\mathbf{S}=\mathbf{S}(\boldsymbol{\eta})$ the second Piola-Kirchhoff tensor and $\mathbf{F}=\mathbf{F}(\boldsymbol{\eta})=\mathbf{I}+\nabla_{0} \boldsymbol{\eta}$ the deformation gradient tensor. We denote by $\mathbf{E}=\mathbf{E}(\boldsymbol{\eta})$ the Green-St Venant strain tensor:

$$
\mathbf{E}=\frac{1}{2}\left(\mathbf{F}^{T} \mathbf{F}-\mathbf{I}\right)=\frac{1}{2}\left(\nabla_{0}^{T} \boldsymbol{\eta}+\nabla_{0} \boldsymbol{\eta}+\nabla_{0}^{T} \boldsymbol{\eta} \nabla_{0} \boldsymbol{\eta}\right)
$$

In this work we consider St Venant-Kirchhoff materials, for which the response function for the second PiolaKirchhoff tensor is linear in $\mathbf{E}$ and given by

$$
\mathbf{S}=\lambda \operatorname{tr}(\mathbf{E}) \mathbf{I}+2 \mu \mathbf{E}
$$

being $\lambda=\frac{E \xi}{(1+\xi)(1-2 \xi)}$ and $\mu=\frac{E}{2(1+\xi)}$ the Lamé constants, with $E$ the Young modulus and $\xi$ the Poisson ratio. Notice that tensor $\mathbf{E}$ is symmetric, implying also the symmetry of the second Piola-Kirchhoff tensor $\mathbf{S}$.

We remark that a 3D linear elastic model is obtained if instead of $\mathbf{E}$ we consider its linear counterpart $\boldsymbol{e}(\boldsymbol{\eta})=\frac{1}{2}\left(\nabla_{0}^{T} \boldsymbol{\eta}+\nabla_{0} \boldsymbol{\eta}\right)$. The result we show in this section can obviously be particularized to this case.

To derive an energy estimate of the FSI problem with such structure model we define the energy of the problem as

$$
\mathscr{E}_{3 D-E}(t)=\frac{\rho}{2}\|\mathbf{u}\|_{L^{2}\left(\Omega^{t}\right)}^{2}+\frac{\rho_{w}}{2}\|\dot{\boldsymbol{\eta}}\|_{L^{2}\left(\Omega_{s}^{0}\right)}^{2}+\mu\|\mathbf{E}\|_{L^{2}\left(\Omega_{s}^{0}\right)}^{2}+\frac{\lambda}{2}\|\operatorname{tr} \mathbf{E}\|_{L^{2}\left(\Omega_{s}^{0}\right)}^{2}
$$

\section{Theorem 3.9.}

(i) Energy decay property: The coupled fluid-structure problem (1), (2), (7), (24) and (25), with homogeneous boundary conditions on $\Gamma_{i n}^{t}$ and $\Gamma_{a}^{t}$, satisfies the following energy equality:

$$
\frac{\mathrm{d}}{\mathrm{d} t}\left(\mathscr{E}_{3 D-E}(t)\right)+2 \nu\|\mathbf{D}(\mathbf{u})\|_{L^{2}\left(\Omega^{t}\right)}^{2}=0
$$

by which we obtain the following energy decay property:

$$
\mathscr{E}_{3 D-E}(T)+2 \nu \int_{0}^{T}\|\mathbf{D}(\mathbf{u})\|_{L^{2}\left(\Omega^{t}\right)}^{2} \mathrm{~d} t=\mathscr{E}_{3 D-E}(0),
$$

where $\mathscr{E}_{3 D-E}(0)$ is a constant depending only on the initial data $\mathbf{u}_{0}, \boldsymbol{\eta}_{0}$ and $\dot{\boldsymbol{\eta}}_{0}$.

(ii) Neumann problem: Under the Hypothesis 3.1, the coupled fluid-structure problem (1), (2), (18), (7), (24) and (25), satisfies the following a priori energy estimate, for all $t \in I$

$$
\mathscr{E}_{3 D-E}(t)+\nu \int_{0}^{t}\|\mathbf{D}(\mathbf{u})\|_{L^{2}(\Omega)}^{2} \mathrm{~d} t \leqslant\left(\mathscr{E}_{3 D-E}(0)+\widetilde{C} \int_{0}^{t}\|\mathbf{r}\|_{L^{2}\left(\Gamma_{i n}^{t}\right)}^{2} \mathrm{~d} t+C \int_{0}^{t}\|\mathbf{q}\|_{L^{2}\left(\Gamma_{a}^{t}\right)}^{2} \mathrm{~d} t\right) \mathrm{e}^{\frac{2 \nu}{\rho} t}
$$


where $\widetilde{C}$ and $C$ are positive constants.

Proof. We proceed as in Theorem 3.4, by multiplying the structure equation (25) by $\dot{\boldsymbol{\eta}}$ and integrating over the reference domain $\Omega_{s}^{0}$.

For the inertia term we obtain

$$
\rho_{w} \int_{\Omega_{s}^{0}} \frac{\partial^{2} \boldsymbol{\eta}}{\partial t^{2}} \cdot \dot{\boldsymbol{\eta}}=\frac{\rho_{w}}{2} \frac{\mathrm{d}}{\mathrm{d} t}\|\dot{\boldsymbol{\eta}}\|_{L^{2}\left(\Omega_{s}^{0}\right)}^{2}
$$

By integration by parts we have that

$$
-\int_{\Omega_{s}^{0}} \operatorname{div}_{0}(\mathbf{P}) \cdot \dot{\boldsymbol{\eta}} \mathrm{d} \omega=-\int_{\partial \Omega_{s}^{0}}\left(\mathbf{P} \cdot \mathbf{n}_{0}\right) \cdot \dot{\boldsymbol{\eta}} \mathrm{d} \gamma+\int_{\Omega_{s}^{0}} \mathbf{P}: \nabla \dot{\boldsymbol{\eta}} \mathrm{d} \omega .
$$

We notice now that, since $\mathbf{S}$ is a symmetric tensor, we have

$$
\mathbf{P}: \nabla \dot{\boldsymbol{\eta}}=\mathbf{F S}: \dot{\mathbf{F}}=\mathbf{S}: \mathbf{F}^{T} \dot{\mathbf{F}}=\mathbf{S}:\left(\frac{1}{2}\left(\mathbf{F}^{T} \dot{\mathbf{F}}+\dot{\mathbf{F}}^{T} \mathbf{F}\right)\right)=\mathbf{S}: \dot{\mathbf{E}} .
$$

So, we can write

$$
\begin{aligned}
\int_{\Omega_{s}^{0}} \mathbf{P}: \nabla \dot{\boldsymbol{\eta}} \mathrm{d} \omega & =\int_{\Omega_{s}^{0}} \mathbf{S}: \dot{\mathbf{E}} \mathrm{d} \omega=\int_{\Omega_{s}^{0}} \lambda \operatorname{tr}(\mathbf{E}) \mathbf{I}: \dot{\mathbf{E}} \mathrm{d} \omega+\int_{\Omega_{s}^{0}} 2 \mu \mathbf{E}: \dot{\mathbf{E}} \mathrm{d} \omega \\
& =\lambda \int_{\Omega_{s}^{0}} \operatorname{tr} \mathbf{E} \operatorname{tr} \dot{\mathbf{E}} \mathrm{d} \omega+\mu \frac{\mathrm{d}}{\mathrm{d} t}\|\mathbf{E}\|_{L^{2}\left(\Omega_{s}^{0}\right)}^{2}=\frac{\lambda}{2} \frac{\mathrm{d}}{\mathrm{d} t}\|\operatorname{tr} \mathbf{E}\|_{L^{2}\left(\Omega_{s}^{0}\right)}^{2}+\mu \frac{\mathrm{d}}{\mathrm{d} t}\|\mathbf{E}\|_{L^{2}\left(\Omega_{s}^{0}\right)}^{2}
\end{aligned}
$$

Regarding the boundary term, due to the boundary conditions of equations (25) and the matching conditions (7) we have that

$$
\int_{\partial \Omega_{s}^{0}}\left(\mathbf{P} \cdot \mathbf{n}_{0}\right) \cdot \dot{\boldsymbol{\eta}} \mathrm{d} \gamma=\int_{\Gamma_{w}^{0}} \widehat{\boldsymbol{\Phi}} \cdot \dot{\boldsymbol{\eta}} \mathrm{d} \gamma=\int_{\Gamma_{w}^{t}} \boldsymbol{\Phi} \cdot \mathbf{u} \mathrm{d} \gamma=-\int_{\Gamma_{w}^{t}}(\boldsymbol{\sigma}(\mathbf{u}, p) \cdot \mathbf{n}) \cdot \mathbf{u} \mathrm{d} \gamma
$$

Thus, we obtain

$$
\frac{\rho_{w}}{2} \frac{\mathrm{d}}{\mathrm{d} t}\|\dot{\boldsymbol{\eta}}\|_{L^{2}\left(\Omega_{s}^{0}\right)}^{2}+\frac{\lambda}{2} \frac{\mathrm{d}}{\mathrm{d} t}\|\operatorname{tr} \mathbf{E}\|_{L^{2}\left(\Omega_{s}^{0}\right)}^{2}+\mu \frac{\mathrm{d}}{\mathrm{d} t}\|\mathbf{E}\|_{L^{2}\left(\Omega_{s}^{0}\right)}^{2}=-\int_{\Gamma_{w}^{t}}(\boldsymbol{\sigma}(\mathbf{u}, p) \cdot \mathbf{n}) \cdot \mathbf{u} \mathrm{d} \gamma
$$

Summing (29) and (15) we derive

$$
\frac{\mathrm{d}}{\mathrm{d} t}\left(\mathscr{E}_{3 D-E}(t)\right)+2 \nu\|\mathbf{D}(\mathbf{u})\|_{L^{2}\left(\Omega^{t}\right)}^{2}=\int_{\Gamma_{i n}^{t} \cup \Gamma_{a}^{t}}\left(\boldsymbol{\sigma}^{t o t}(\mathbf{u}, p) \cdot \mathbf{n}\right) \cdot \mathbf{u} \mathrm{d} \gamma
$$

which leads to the energy decay property by taking homogeneous boundary conditions on $\Gamma_{i n}^{t}$ and $\Gamma_{a}^{t}$, and to the desired inequality, by bounding from above its right hand side as in Theorem 3.6.

We conclude this section by observing that the 3D elastic model, like the models presented in Section 3.1, does not provide sufficient regularity for $\boldsymbol{\eta}$ to be coupled with the Navier-Stokes equations. However, for this problem recent well posedness results for strong solutions have been obtained in [8].

\section{The 1D MOdeL}

One-dimensional (1D) models can be derived from the incompressible Navier-Stokes equations coupled with a structure model for the vessel wall, by making some simplifying assumptions and integrating over the cross section of the artery (see $[13,14]$ ). The vessel is assumed to have axial symmetry and fixed cylinder axis. 
Furthermore only radial displacements are considered and dominance of the axial velocity is supposed. Finally it is assumed that the pressure is constant on each transversal section and that there are no body forces. Under these assumptions, and assuming a specific axial velocity profile, the 1D model for blood flow in a cylindrical vessel is given by the following system:

$$
\left\{\begin{array}{rl}
\frac{\partial A}{\partial t}+\frac{\partial Q}{\partial z} & =0 \\
\frac{\partial Q}{\partial t}+\frac{\partial}{\partial z}\left(\frac{\alpha Q^{2}}{A}\right)+\frac{A}{\rho} \frac{\partial \bar{p}}{\partial z} & =-2 \pi \nu(\alpha+2) \frac{Q}{A}
\end{array} \quad z \in(a, b), t \in I .\right.
$$

Here $(b-a)$ is the vessel length, $z$ denotes the axial direction, $\alpha$ is the momentum flux correction coefficient, $\nu$ is the fluid dynamic viscosity and $\rho$ is the fluid density; $\alpha, \nu$ and $\rho$ are assumed constant and we take $\alpha=1$, corresponding to a flat velocity profile. The unknowns are the cross-section area $A$, the flow rate $Q$ and the averaged pressure $\bar{p}$.

System (31) is closed by providing an algebraic relation linking pressure and area. We consider here the same pressure-area algebraic relation (11) mentioned in Section 2. Written in terms of $\bar{p}$ and $A$ it becomes

$$
\psi\left(A ; A_{0}, \beta\right)=\beta \frac{\sqrt{A}-\sqrt{A_{0}}}{A_{0}} \quad \text { with } \quad \beta=\frac{\sqrt{\pi} h_{0} E}{1-\xi^{2}} .
$$

Both $E$ and $A_{0}$ may be functions of $z$.

With this closing pressure-area algebraic relation system (31) turns out to be hyperbolic and possesses two distinct eigenvalues (see [13]) $\lambda_{1,2}=\bar{u} \pm \sqrt{\frac{\beta}{2 \rho A_{0}}} A^{\frac{1}{4}}$. Their corresponding eigenfunctions (characteristic variables) are given by

$$
W_{1,2}=\bar{u} \pm 4 \sqrt{\frac{\beta}{2 \rho A_{0}}}\left(A^{\frac{1}{4}}-A_{0}^{\frac{1}{4}}\right)
$$

Under physiological conditions for haemodynamics the flow is subcritical (see [13]), i.e., the eigenvalues $\lambda_{1}$ and $\lambda_{2}$ have opposite signs: $\lambda_{1}>0$ and $\lambda_{2}<0$. In this case $W_{1}$ corresponds to the incoming characteristic on the entering point $z=a$, while $W_{2}$ is the incoming characteristic on the exiting point $z=b$.

We take the following initial and boundary conditions

$$
\begin{array}{ll}
A(0, z)=A^{0}, & Q(0, z)=Q^{0}, \quad a \leqslant z \leqslant b \\
W_{1}(t)=g_{1}(t), & z=a \\
W_{2}(t)=g_{2}(t), & z=b
\end{array}
$$

where $g_{1}$ and $g_{2}$ are given functions, and define the energy of the $1 \mathrm{D}$ model as

$$
\mathscr{E}_{1 D}(t):=\frac{\rho}{2} \int_{a}^{b} A(t, z) \bar{u}^{2}(t, z) \mathrm{d} z+\int_{a}^{b} \Psi(A(t, z)) \mathrm{d} z, \quad \forall t \in I, \quad \text { with } \quad \Psi(A)=\int_{A_{0}}^{A} \psi(\tau) \mathrm{d} \tau
$$

It was shown in [9] that, for $\psi(t)$ given by relation $(32), \Psi(A)$ is always positive, making $\mathscr{E} 1 D(t)$ a positive function for all $Q, A>0$ and $t$. The next result holds (see [9]).

Lemma 4.1. System (31) together with the pressure-area relation (32) satisfies the following energy equality:

$$
\frac{\mathrm{d}}{\mathrm{d} t}\left(\mathscr{E}_{1 D}(t)\right)+K_{r} \rho \int_{a}^{b} \bar{u}^{2} \mathrm{~d} z+\left.Q\left(\bar{p}+\frac{\rho}{2} \bar{u}^{2}\right)\right|_{a} ^{b}=0
$$




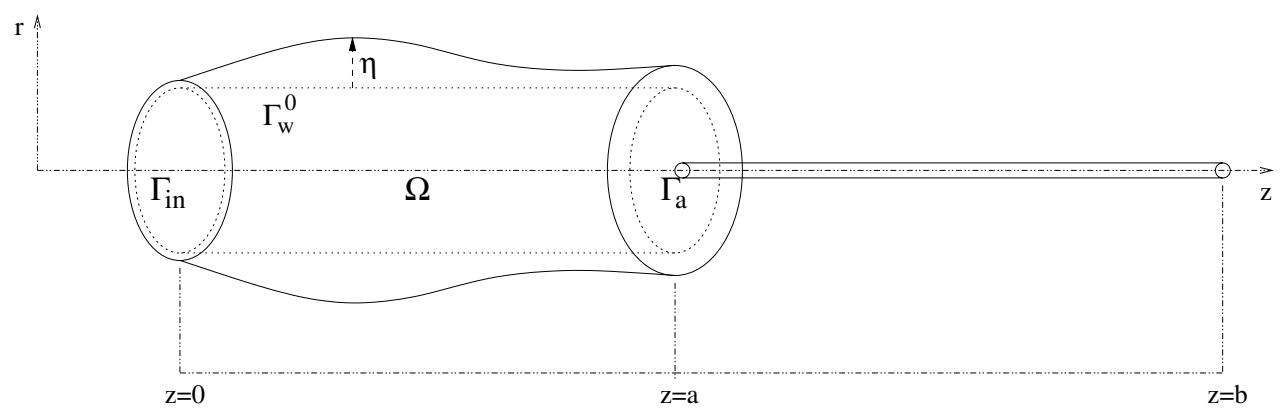

Figure 4. Cylindrical vascular region $\Omega$.

which leads to the following energy conservation property:

$$
\mathscr{E}_{1 D}(T)+K_{r} \rho \int_{0}^{T} \int_{a}^{b} \bar{u}^{2} \mathrm{~d} z \mathrm{~d} t+\left.\int_{0}^{T} Q\left(\bar{p}+\frac{\rho}{2} \bar{u}^{2}\right)\right|_{a} ^{b} \mathrm{~d} t=\mathscr{E}_{1 D}(0) .
$$

Furthermore, we have that

(i) if homogeneous boundary conditions are considered $\left(g_{1}(t)=g_{2}(t)=0, \forall t \in I\right)$, then

$$
\mathscr{E}_{1 D}(T)+K_{r} \rho \int_{0}^{T} \int_{a}^{b} \bar{u}^{2} \mathrm{~d} z \mathrm{~d} t \leqslant \mathscr{E}_{1 D}(0)
$$

(ii) if the boundary data satisfies

$$
g_{1}(t)>-4 \sqrt{\frac{\beta}{2 \rho A_{0}}} A^{\frac{1}{4}}, \quad \text { and } \quad g_{2}(t)<4 \sqrt{\frac{\beta}{2 \rho A_{0}}} A^{\frac{1}{4}}, \quad \forall t \in I \text {, }
$$

then there exists a positive function $G\left(g_{1}, g_{2}\right)$ such that

$$
\mathscr{E}_{1 D}(T)+K_{r} \rho \int_{0}^{T} \int_{a}^{b} \bar{u}^{2} \mathrm{~d} z \mathrm{~d} t \leqslant \mathscr{E}_{1 D}(0)+\int_{0}^{T} G\left(g_{1}(t), g_{2}(t)\right) \mathrm{d} t .
$$

\section{Coupling the 3D with the 1D Model}

In this section we study the 3D-1D FSI coupling problem defined by the 3D Navier-Stokes equations (1) and (2), the matching conditions between the 3D fluid and the vessel wall (7), the structure model (10) and (22) or (25) and (24), and the 1D model (31)-(33), as a whole. By putting together the 3D FSI energy estimates derived in Section 3 and the 1D energy estimate provided in Section 4, we will be able to deduce the proper matching conditions to impose at the interface between the $3 \mathrm{D}$ and $1 \mathrm{D}$ FSI models.

We consider the 3D domain of Figure 1 coupled with a $1 \mathrm{D}$ domain $I=(a, b)$ (see Fig. 4 ). On the first domain we solve the $3 \mathrm{D}$ fluid-structure interaction problem while in the second we take the simplified 1D model.

At the exit point $z=b$ we consider an absorbing boundary condition $W_{2}=0$, which means that the incoming characteristic vanishes. If the structure model on the $3 \mathrm{D}$ problem requires boundary conditions we take Dirichlet homogeneous boundary conditions for the structure at the inlet $\left(\Gamma_{w}^{t} \cap \Gamma_{i n}^{t}\right.$ or $\Gamma_{s, i n}^{t}$, according to the model).

The objective is to find the proper interface conditions we need to impose on $\Gamma_{a}^{t}$ such that the 3D-1D FSI coupled problem is stable. At the interface $z=a$ the $1 \mathrm{D}$ model provides the averaged quantities $Q_{1 D}, \bar{p}_{1 D}$, 
$A_{1 D}$ and $\bar{u}_{1 D}=\frac{Q_{1 D}}{A_{1 D}}$. These quantities are defined in the $3 \mathrm{D}$ model, for the same interface $\Gamma_{a}^{t}$ as:

$$
Q_{3 D}=\int_{\Gamma_{a}^{t}} \mathbf{u} \cdot \mathbf{n d} \gamma, \quad A_{3 D}=\left|\Gamma_{a}^{t}\right|, \quad \bar{p}_{3 D}=\frac{1}{\left|\Gamma_{a}^{t}\right|} \int_{\Gamma_{a}^{t}} p \mathrm{~d} \gamma, \quad \bar{u}_{3 D}=\frac{Q_{3 D}}{\left|\Gamma_{a}^{t}\right|}
$$

By imposing the continuity of all or some of the above mentioned quantities (or functions of them) at the interface $\Gamma_{a}^{t}$, we will be able to provide the $3 \mathrm{D}$ model with averaged boundary data on $\Gamma_{a}^{t}$. However, the $3 \mathrm{D}$ Navier-Stokes equations require pointwise data and thus these averaged quantities have to be properly set up. There are two possibilities: either the 3D model is provided with a mean pressure type of data (mean pressure problem), or with a flow rate one (flow rate problem).

For the mean pressure problem, Heywood et al. [24] proposed an approach resulting in imposing a constant normal stress on $\Gamma_{a}^{t}$. For the case of our Navier-Stokes formulation (1), this approach consists in considering the normal component of the total stress to be constant and equal to the given total mean pressure value.

Regarding the flow rate problem we follow the Lagrange multiplier approach proposed in $[15,40]$. It consists in considering the flux condition as a constraint imposed in a weak sense through a Lagrange multiplier. Also in this case, for the formulation of the fluid equations here presented, the normal component of the total stress, although unknown, turns out to be constant.

We observe that the imposition of defective boundary data for the Navier-Stokes problem is subject of active research. In particular, well posedness results for the two above mentioned cases were given in the cited works only for the rigid wall case.

In order to derive an a priori energy estimate, we start by taking the simplest structure model on the $3 \mathrm{D}$ problem, that is the pressure-area algebraic relation (11). By summing the energy inequalities obtained separately for the 3D FSI model (12) and the 1D one (34), we obtain

$\frac{\mathrm{d}}{\mathrm{d} t}\left(\mathscr{E}_{3 D-A L}(t)\right)+2 \nu\|\mathbf{D}(\mathbf{u})\|_{L^{2}\left(\Omega^{t}\right)}^{2}+\frac{\mathrm{d}}{\mathrm{d} t}\left(\mathscr{E}_{1 D}(t)\right)+K_{r} \rho \int_{a}^{b} \bar{u}^{2} \mathrm{~d} z=\int_{\Gamma_{i n}^{t} \cup \Gamma_{a}^{t}}\left(\boldsymbol{\sigma}^{t o t}(\mathbf{u}, p) \cdot \mathbf{n}\right) \cdot \mathbf{u} \mathrm{d} \gamma-\left.Q\left(\bar{p}+\frac{\rho}{2} \bar{u}^{2}\right)\right|_{a} ^{b}$

Supposing that we are able to bound from above in terms of the problem data the boundary terms not corresponding to the interface section, for instance as it was done in Theorem 3.6, we easily verify from (37) that an energy estimate can be achieved if the coupling terms on $\Gamma_{a}^{t}$ due to the 3D fluid equations and the 1D model cancel out. To that purpose we define as interface conditions on $\Gamma_{a}^{t}$ the continuity of the normal component of the total stress $\boldsymbol{\sigma}^{\text {tot }}$ and of the flux $Q$ :

$$
\left\{\begin{aligned}
-\boldsymbol{\sigma}^{\text {tot }} \cdot \mathbf{n}=p \mathbf{n}+\frac{\rho}{2}|\mathbf{u}|^{2} \mathbf{n}-2 \nu \mathbf{D}(\mathbf{u}) \cdot \mathbf{n} & =\left(\bar{p}_{1 D}+\frac{\rho}{2}\left|\bar{u}_{1 D}\right|^{2}\right) \mathbf{n}, & & \text { on } \Gamma_{a}^{t} \\
Q_{3 D}=\int_{\Gamma_{a}^{t}} \mathbf{u} \cdot \mathbf{n} \mathrm{d} \gamma & =Q_{1 D}, & & \text { on } \Gamma_{a}^{t} .
\end{aligned}\right.
$$

We now notice that, from the interface conditions (38), and the methods described above to treat the defective boundary conditions on the $3 \mathrm{D}$ problem, we have

$$
\int_{\Gamma_{a}^{t}}\left(p \mathbf{n}+\frac{\rho}{2}|\mathbf{u}|^{2} \mathbf{n}-2 \nu \mathbf{D}(\mathbf{u}) \cdot \mathbf{n}\right) \cdot \mathbf{u} \mathrm{d} \gamma=\left(\bar{p}_{1 D}+\frac{\rho}{2}\left|\bar{u}_{1 D}\right|^{2}\right) \int_{\Gamma_{a}^{t}} \mathbf{u} \cdot \mathbf{n} \mathrm{d} \gamma=Q_{1 D}\left(\bar{p}_{1 D}+\frac{\rho}{2}\left|\bar{u}_{1 D}\right|^{2}\right)
$$

which makes the right hand side of (37) to vanish. So we can conclude the following result.

Theorem 5.1. Consider the 3D-1D FSI coupling problem, given by (1), (2), (7), (11) and (31)-(33), together with the coupling conditions (38). We have the following cases.

(i) Energy decay property: if homogeneous boundary conditions are taken on $\Gamma_{\text {in }}^{t}$, and absorbing boundary conditions are considered for the $1 D$ problem in $z=b$, then the problem satisfies the following energy 
decay property:

$$
\mathscr{E}_{3 D-A L}(T)+2 \nu \int_{0}^{T}\|\mathbf{D}(\mathbf{u})\|_{L^{2}\left(\Omega^{t}\right)}^{2} \mathrm{~d} t+\mathscr{E}_{1 D}(T)+K_{r} \rho \int_{0}^{T} \int_{a}^{b} \bar{u}^{2} \mathrm{~d} z \mathrm{~d} t \leqslant \mathscr{E}_{3 D-A L}(0)+\mathscr{E}_{1 D}(0)
$$

(ii) Neumann problem: if a Neumann boundary condition (18) is considered on $\Gamma_{i n}^{t}$, and a non homogeneous boundary condition is considered for the $1 D$ problem at $z=b$ then the problem satisfies the following a priori energy estimate, for all $t \in I$

$$
\begin{aligned}
\mathscr{E}_{3 D-A L}(t)+\nu & \int_{0}^{t}\|\mathbf{D}(\mathbf{u})\|_{L^{2}(\Omega)}^{2} \mathrm{~d} t+\mathscr{E}_{1 D}(t)+K_{r} \rho \int_{0}^{t} \int_{a}^{b} \bar{u}^{2} \mathrm{~d} z \mathrm{~d} t \\
& \leqslant\left(\mathscr{E}_{3 D-A L}(0)+\mathscr{E}_{1 D}(0)+\widetilde{C} \int_{0}^{t}\|\mathbf{r}\|_{L^{2}\left(\Gamma_{i n}^{t}\right)}^{2} \mathrm{~d} t+\int_{0}^{t} G\left(g_{2}(t)\right) \mathrm{d} t\right) \mathrm{e}^{\frac{2 \nu}{\rho} t}
\end{aligned}
$$

where $G$ is as defined in Lemma 4.1, $\widetilde{C}$ is a positive constant as defined in Theorem 3.6 and $\mathscr{E}_{3 D-A L}(0)$ and $\mathscr{E}_{1 D}(0)$ depend only on the initial conditions.

Remark 5.2. The results are readily extended to the case of more than one interface section, by applying conditions (38) to each section separately, keeping in mind the sign conventions for the flux. Clearly if all artificial sections are interfaced with the 1D model, we can apply directly estimate (39). In this case, an extra positive term, depending on the boundary data of the 1D model, will appear on the right hand side of (39), as in (36) (see Lem. 4.1).

Remark 5.3. To impose interface conditions (38) in practice (or any linear combination of them) to the 3D-1D coupled problem, we will associate one of them to the 3D FSI subproblem, eventually understood as a "defective condition" $[15,24,40]$, and the other one to the 1D subproblem. In particular, for the prescription of either the flux or the total pressure at the 1D interface point, we follow the approach described in [13], consisting in combining the boundary datum with the compatibility condition associated to the outgoing characteristic variable.

If, instead of the pressure-area algebraic relation for the structure, we use the independent rings model (21), exactly the same result can be deduced, replacing $\mathscr{E}_{3 D-A L}$ with $\mathscr{E}_{3 D-I R}$ and noticing that $\mathscr{E}_{3 D-I R}(0)$ depends also on $\eta_{0}$ and $\dot{\eta}_{0}$.

If the generalized strings model (23) is considered instead, boundary conditions have to be prescribed on the displacement $\eta_{r}$ at $\partial \Gamma_{w}^{t}=\left(\Gamma_{w}^{t} \cap \Gamma_{i n}^{t}\right) \cup\left(\Gamma_{w}^{t} \cap \Gamma_{a}^{t}\right)$. In fact, this boundary term appears in the final energy estimate of the coupled 3D-1D problem. For instance, if a homogeneous boundary condition is prescribed on the Navier-Stokes equations at $\Gamma_{i n}^{t}$, we have the following inequality:

$$
\begin{aligned}
\mathscr{E}_{3 D-G S}(T)+ & 2 \nu \int_{0}^{T}\|\mathbf{D}(\mathbf{u})\|_{L^{2}\left(\Omega^{t}\right)}^{2} \mathrm{~d} t+\mathscr{E}_{1 D}(T)+K_{r} \rho \int_{0}^{T} \int_{a}^{b} \bar{u}^{2} \mathrm{~d} z \mathrm{~d} t \\
& \leqslant \mathscr{E}_{3 D-G S}(0)+\mathscr{E}_{1 D}(0)+\int_{0}^{T} G\left(g_{2}(t)\right) \mathrm{d} t+\int_{0}^{T} \int_{\Gamma_{w}^{0} \cap \Gamma_{a}^{0}} a \frac{\partial \eta_{r}}{\partial z} \frac{\partial \eta_{r}}{\partial t} \mathrm{~d} \gamma \mathrm{d} t .
\end{aligned}
$$

Since the 1D model employs only an algebraic structure model, and therefore does not require any boundary condition of the wall displacement, the boundary term appearing in (40) can not be balanced with the corresponding 1D term. As it was shown in Theorem 5.1, an energy inequality can be easily derived if homogeneous boundary conditions are chosen for the 3D wall displacement. Yet, this choice does not guarantee that the vessel cross-section area is continuous between the 3D and 1D models. We will see that this drawback does not seem to be relevant in numerical simulations, since the coupling carried out without imposing the continuity of the area at the interface works very well. 
On the other hand, we may include the continuity of the cross-section area as a further coupling condition on $\Gamma_{a}^{t}$ between the two models. This resorts in imposing non homogeneous Dirichlet boundary conditions to the $3 \mathrm{D}$ vessel displacement. Yet, it is not obvious that imposing the continuity of the area at the interface $\Gamma_{a}^{t}$ will yield the stability of the coupled problem, since this does not necessarily imply a bound on the boundary term of (40).

We remark that the issue of the continuity of the area at the coupling interface did not come up with the simpler algebraic law and independent rings models because they intrinsically admit discontinuities on the displacement $\eta_{r}$ and hence on the area. Nevertheless, also these models have proved to work very well at the numerical level.

We finish this discussion by noticing that imposing the continuity of the flux as in (38) actually guarantees the conservation of the mass, even when the continuity of the area is not assured.

Finally, the same result on the energy estimate of the 3D-1D coupled problem can be obtained if we consider the 3D non-linear elastic model (25) for the description of the wall movements. In this case, as for the generalized strings model, a boundary term appears in the energy inequality and the energy estimate can be deduced by imposing homogeneous boundary conditions at $\Gamma_{s, a}^{0}$ (as for instance in (25)). The same considerations made for the generalized strings model apply here.

\section{Numerical RESULtS}

The numerical approximation of the 3D-1D FSI coupling is carried out considering two sub-problems, the 3D and the 1D, separately. Here, we solve the coupling explicitly, meaning that there are no sub-iterations at each time step. The coupling strategy applied consists in passing, at each time step, the flux computed by the $3 \mathrm{D}$ solver at the previous time step to the 1D solver, which, in turn, computes the total pressure to be fed into the 3D model.

The Navier-Stokes equations are discretized in time by the implicit Euler scheme, combined with an Arbitrary Lagrangian Eulerian formulation to account for the moving boundary. Whenever the structure model includes an inertia term, the time discretization of the structure is done through a Newmark scheme. The space discretization of the fluid is done using $P 1-P 1 b u b l e$ finite elements, while for the structure we use $P 1$ finite elements. The fluid-structure coupling is solved implicitly through a quasi-Newton algorithm [11], where in the fluid tangent operator we have neglected the shape derivatives (i.e. the derivatives with respect to the position of the moving boundary). The fluid normal stress on the fluid-structure interface has been computed in a weak form evaluating the residual of the fluid equation for the non vanishing test functions on the interface. Observe however that, with the new formulation adopted, such residual represents the normal total stress, and it has to be corrected by subtracting the computed quantity $\frac{\rho}{2}|\mathbf{u}|^{2}$. Finally, we have used a conforming finite element space discretization between fluid and structure. The 1D problem is solved through a Lax-Wendroff finite element scheme [13], which has very good dispersion error properties. All the numerical solvers have been developed in $\mathrm{C}++$ within the finite element library LifeV (http://www.lifev.org/).

We consider a straight cylindrical vessel of length $5 \mathrm{~cm}$, with radius of $0.5 \mathrm{~cm}$ and wall thickness of $0.1 \mathrm{~cm}$. We take the fluid viscosity $\nu=0.03$ poise and the fluid density $\rho=1 \mathrm{~g} \cdot \mathrm{cm}^{-3}$. Regarding the structure properties, we take the density $\rho_{w}=1.2 \mathrm{~g} \cdot \mathrm{cm}^{-3}$, the Poisson ratio $\xi=0.3$ and the Young Modulus $E=3 \times 10^{6}$ dyne $\cdot \mathrm{cm}^{-2}$. A pressure of $1.3332 \times 10^{4}$ dyne. $\mathrm{cm}^{-2}(100 \mathrm{~mm} \mathrm{Hg})$ is imposed at the inlet $\Gamma_{\text {in }}^{t}$ during $3 \times 10^{-3} \mathrm{~s}$, representing a pressure impulse. Although a single impulse is not a realistic condition for the application at hand, it is useful to highlight some properties of the coupling, in particular the presence of spurious reflections.

We couple the 3D fluid structure-interaction model with a $1 \mathrm{D}$ model of length $5 \mathrm{~cm}$ in which we considered 50 finite elements. The 3D domain is composed of 2880 elements in the fluid domain and 3840 elements in the solid domain (when using the 3D elastic model for the structure). We carry out the simulations using a time step of $0.25 \times 10^{-3} \mathrm{~s}$ in the $3 \mathrm{D}$ code, and $10^{-5} \mathrm{~s}$ in the $1 \mathrm{D}$ code. The results of this $3 \mathrm{D}-1 \mathrm{D}$ coupling are compared with the solution of the 3D fluid-structure interaction problem in a vessel with the same characteristics but with double length $(10 \mathrm{~cm})$, which is considered the "exact solution". The final time is $T=0.02 \mathrm{~s}$, which is sufficient in our test for the wave to leave the computational domain. For the "exact solution" problem we consider the 


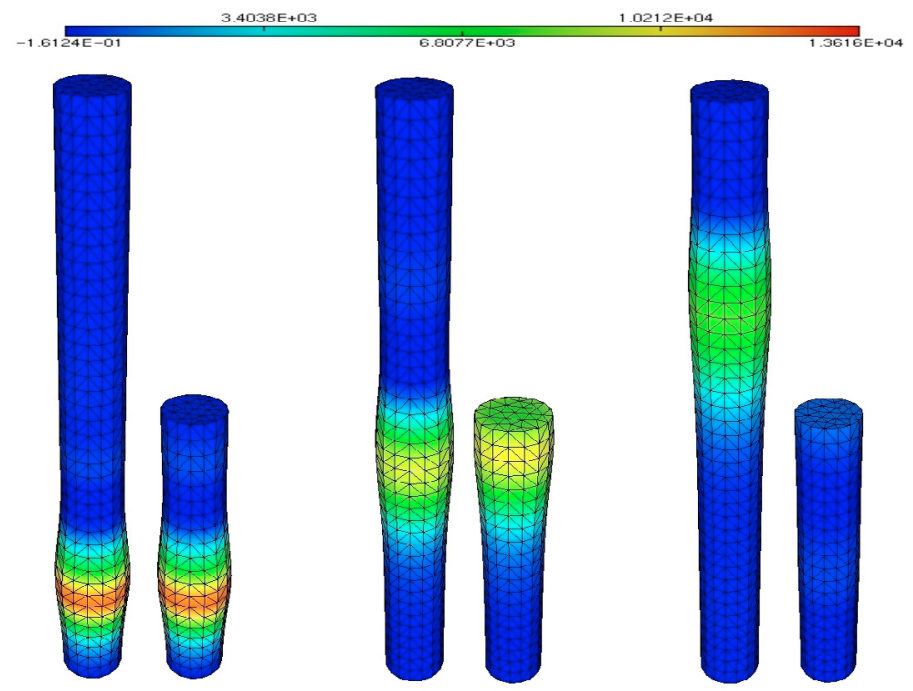

Figure 5. Comparison between the pressure values of the exact and the coupled solutions at three different times $(t=0.005,0.01$ and 0.015$)$, using a $3 \mathrm{D}$ linear elastic model for the structure, without imposing the continuity of the area at the coupling interface.

same boundary conditions as for the coupling problem at the inflow, and we impose a zero Neumann condition at outflow for the fluid. For the structure we use at outflow boundary conditions as in (25). Notice that these are reflecting boundary conditions, nevertheless, for the time interval we are considering the wave does not reach the end of the tube.

We consider four different test cases in this geometry, corresponding to three different structure models for the vessel wall: the pressure-area algebraic law, the independent rings model, and a 3D linear elastic model. For the 3D linear elastic model two situations are treated: in one we do not impose the continuity of the area at the coupling interface $\Gamma_{a}^{t}$, leaving the structure free to move radially at that interface by prescribing the homogeneous boundary conditions described in Section 3; in the other we force the continuity of the area, by imposing the displacement provided by the $1 \mathrm{D}$ model through relation (32) on the 3D structure, by means of a Dirichlet boundary condition. From the value of the area coming from the $1 \mathrm{D}$ problem $A^{1 D}$ we evaluate the displacement to prescribe at each point of $\Gamma_{w}^{t} \cap \Gamma_{a}^{t}$ assuming that the movement of $\Gamma_{w}^{t} \cap \Gamma_{a}^{t}$ are only radial and that its shape does not change in time. That is, we set

$$
\boldsymbol{\eta}=\frac{\sqrt{A^{1 D}}-\sqrt{A_{0}^{3 D}}}{\sqrt{A_{0}^{3 D}}} \hat{\mathbf{x}}, \quad \text { on } \Gamma_{a}^{t} \cap \Gamma_{w}^{t}
$$

where $A_{0}^{3 D}$ is the area of the section $\Gamma_{a}^{0}$.

In Figure 5 we show the pressure values of the "exact" and the coupled solutions at three different times, using the 3D linear elastic model without imposing the continuity of the area. It is evident that, even without imposing the continuity of the area at the coupling interface, the 1D model acts as an absorbing boundary condition, eliminating the spurious reflections.

To study the influence of the different structural models here considered, we analyze the time evolution of the solution on two different sections of the cylinder: the section in middle of the tube, at $z=2.5 \mathrm{~cm}$, and the ending section, interfacing with the $1 \mathrm{D}$ model, at $z=5 \mathrm{~cm}$. Figures $6-8$ show the comparison between the "exact" and coupled solution values of the area, mean pressure and flux, respectively, in the two sections, for the four cases studied. We verify that, whatever structural model is employed, the coupled solution is in good agreement with the "exact" one, except for the case where the continuity of the area is forced. In that case, the 

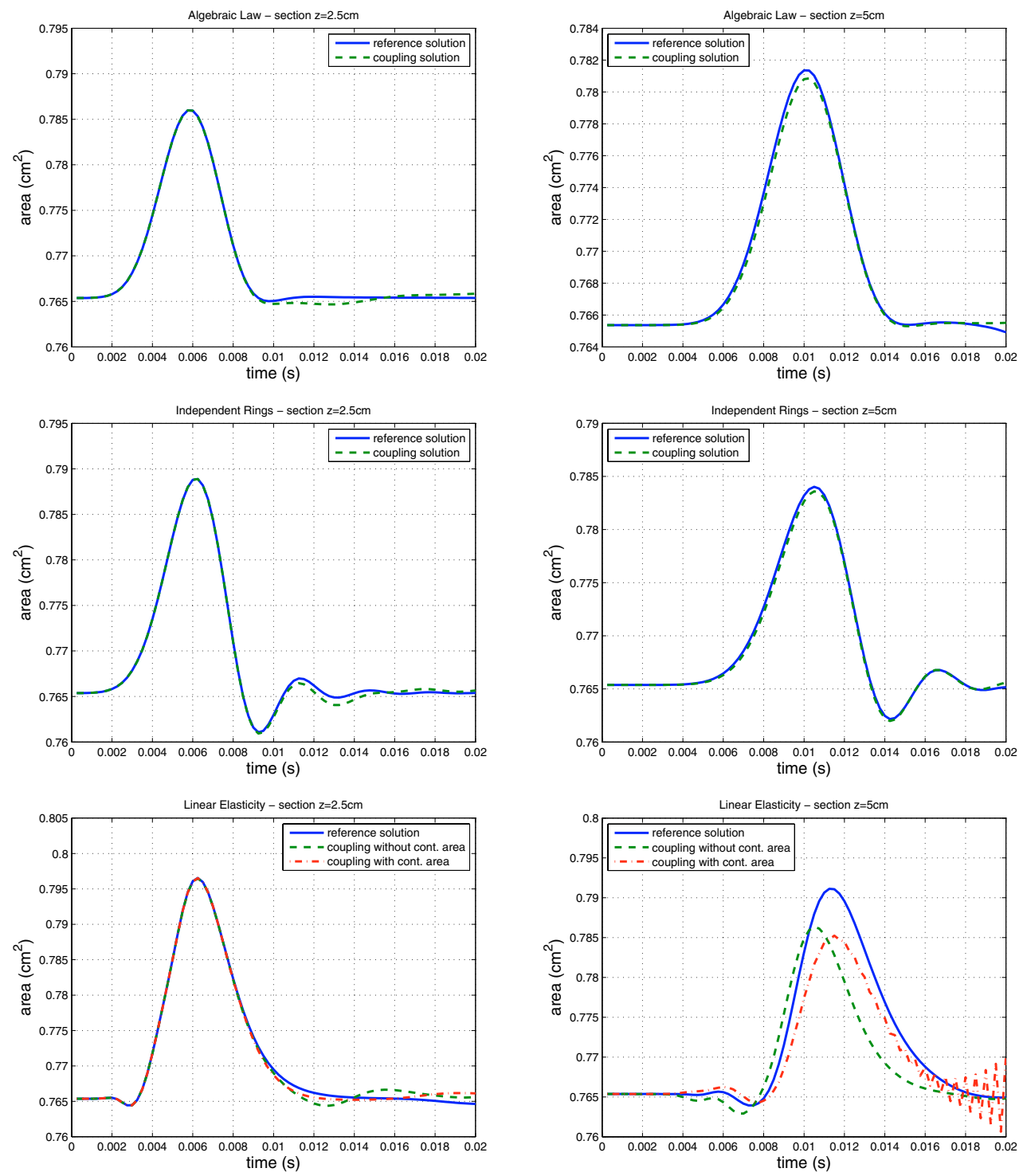

FiguRE 6. Comparison between exact and coupled solution values of the area, for the four test cases considered, at two sections: the middle one $z=2.5 \mathrm{~cm}$ (left) and the interface section with the $1 \mathrm{D}$ model $z=5 \mathrm{~cm}$ (right).

coupling seems to work very well until when instabilities start to appear at the coupling interface, where the area is imposed. We recall that, in this case, a stability result could not be obtained, and the numerical results seem to confirm this fact. We wish however to point out that the study of the stability of numerical algorithms for the coupling is not the subject of the present paper, and will be addressed in a forthcoming work. It may be possible that by using an implicit coupling algorithm the continuity of the area could be more robustly imposed. Nevertheless, we remark once more how the coupling without imposition of the continuity of the area proves to work very well.

These considerations are confirmed when looking at the relative errors on the main quantities (namely area, mean pressure and flux), presented in Figure 9. The relative errors are computed as the difference between 

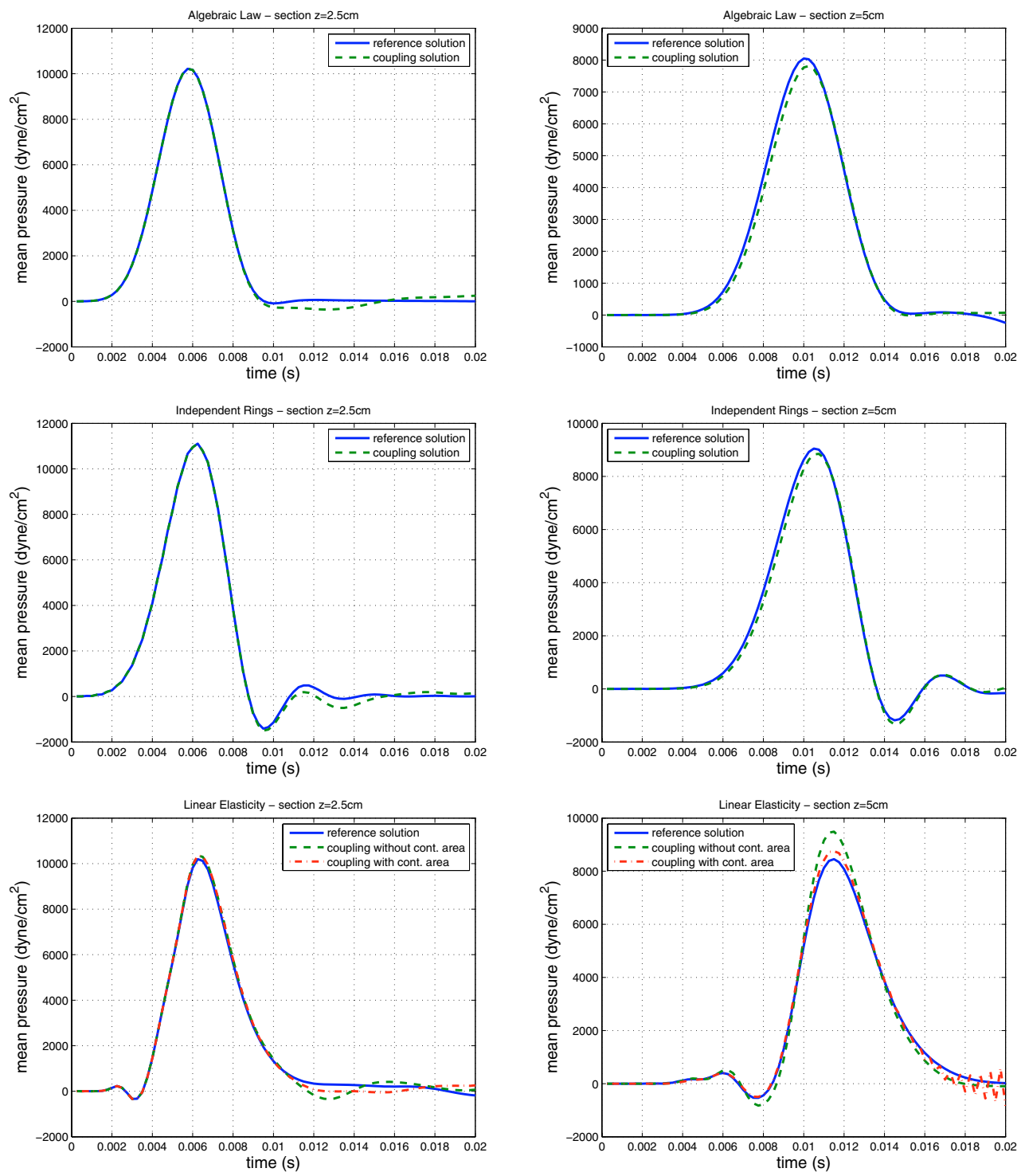

Figure 7. Comparison between exact and coupled solution values of the mean pressure, for the four test cases considered, at two sections: the middle one $z=2.5 \mathrm{~cm}$ (left) and the interface section with the $1 \mathrm{D}$ model $z=5 \mathrm{~cm}$ (right).

the quantity computed by the 3D-1D coupled model and the corresponding "exact" quantity, divided by the maximum value in time. Observe that, even at the coupling interface, where the maximum relative errors are found, the errors of the mean pressure and the area remain under $10 \%$, except in the case where the continuity of the area is imposed. The errors of the flux at the coupling interface are slightly higher due to the fact that we are performing an explicit coupling, and the flux at the coupling interface is in fact the one at the previous time step. We have carried out some numerical tests for different values of the time step, which have demonstrated that these relative errors remain roughly the same. This shows that the errors plotted in Figure 9 are actually due to the 3D-1D coupling (modelling errors), and they are not affected by the time discretization scheme. We are currently investigating the use of implicit algorithms based on fixed point iterations. The results obtained so 

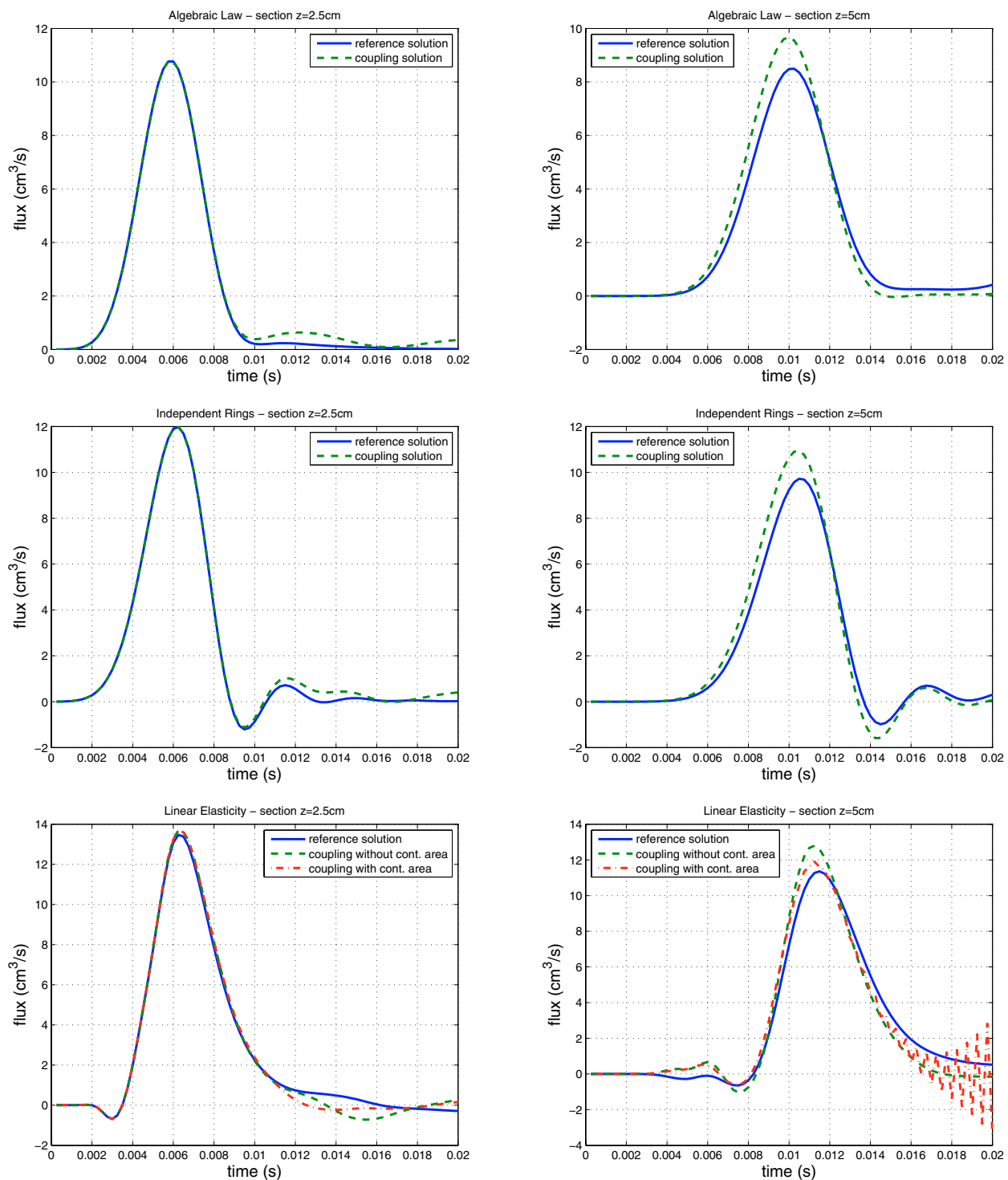

Figure 8. Comparison between exact and coupled solution values of the flux, for the four test cases considered, at two sections: the middle one $z=2.5 \mathrm{~cm}$ (left) and the interface section with the $1 \mathrm{D}$ model $z=5 \mathrm{~cm}$ (right).

far show a similar pattern and magnitude for the errors. More details can be found in [27]. We also remark the much improved quality of these results compared with those obtained by using standard boundary conditions, like for instance stress free conditions at the artificial sections, in which the relative errors are of the order of $75 \%$.

Finally, in the picture of Figure 10 we present the discrepancies of the area between the 3D and 1D models at the coupling interface. We compute the relative differences on the area as $\frac{A_{3 D}-A_{1 D}}{A_{0}}$, where $A_{0}$ is the reference area, which is in our case $\pi R_{0}^{2}=0.7854 \mathrm{~cm}^{2}$. Obviously, in the case where the continuity of the area 

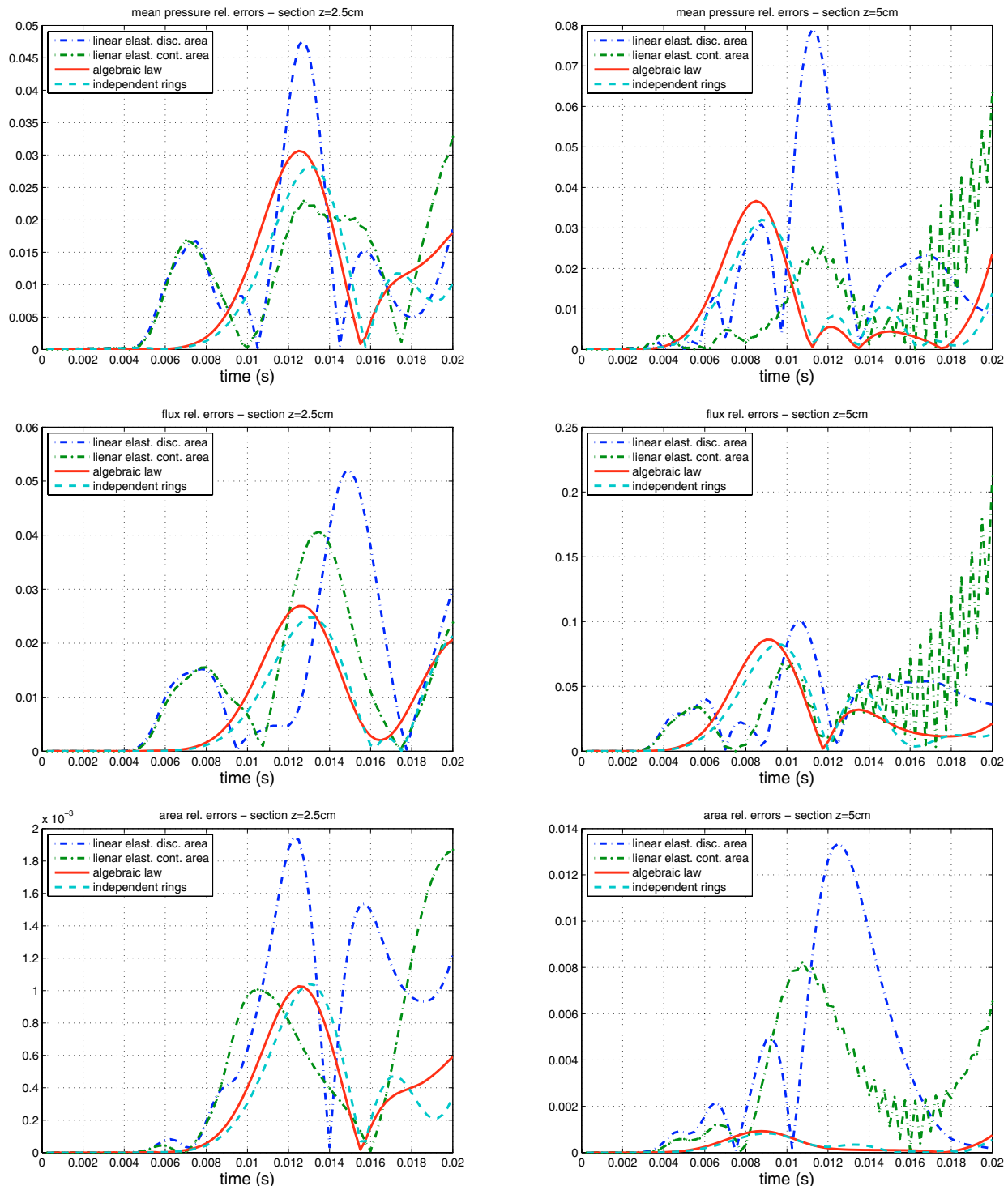

Figure 9. Relative errors for the solution values of the mean pressure (up), the flux (middle) and the area (bottom), for the four test cases considered, at two sections: the middle one $z=2.5 \mathrm{~cm}$ (left) and the interface section with the 1D model $z=5 \mathrm{~cm}$ (right).

is forced at the interface, that difference is zero. In all other cases discontinuities of the area are expected, yet they are bounded and rather small.

\section{Conclusions}

We have considered the coupling between 3D and 1D fluid-structure interaction models describing the flow inside compliant tubes. We proposed a 3D-1D coupling based on the continuity, at the coupling interface, of the normal total stress and of the flux. By adopting a reformulation of the Navier-Stokes equations, for which the natural boundary condition of the weak problem is precisely the normal total stress, we derived an a priori 


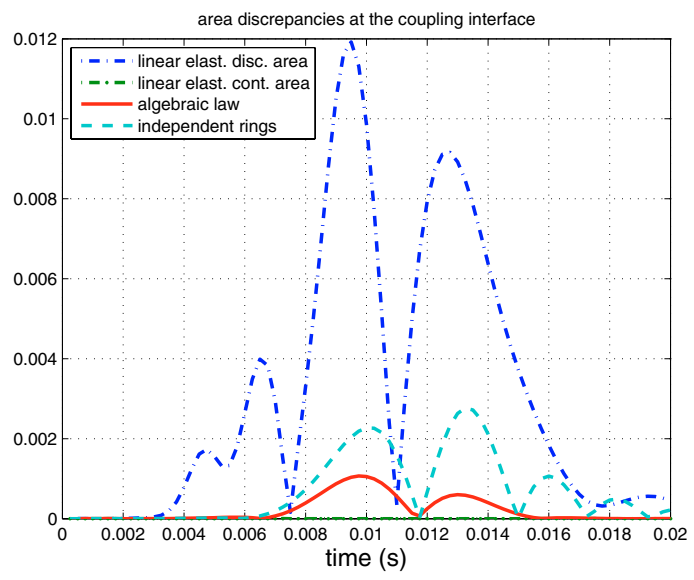

Figure 10. Relative difference between the 3D and the 1D areas at the coupling interface, for the four test cases considered.

energy estimate for the global 3D-1D FSI coupling. Several models for the structure vessel wall on the 3D problem were considered, starting with a simple algebraic model and concluding with a 3D non-linear elastic one, and the energy estimate result of the 3D-1D model was extended to each case.

We have shown that if the structure wall model does not require boundary conditions, the stability result is readily obtained. On the other hand, if the structure model requires boundary conditions, the stability result is obtained only by choosing homogeneous (either Dirichlet or Neumann) boundary conditions for the structure. In both cases, the stability of the 3D-1D FSI coupling is obtained without imposing the continuity of the area at the coupling interface. Nevertheless, the prescription of the continuity of the flux guarantees the conservation of the mass, so that the coupling is consistent.

Intensive numerical tests were carried out, showing that the coupling works very well, even for an explicit coupling algorithm, which was the strategy here applied.

The use of the proposed non standard Navier-Stokes formulation has been essential to obtain the stability results. We note, however, that for the test cases presented here, where the term $\frac{\rho}{2}|\mathbf{u}|^{2}$ is small compared to the pressure, the use of the standard Navier-Stokes equations may work equally well in practice, as shown in [9]. Whenever $\frac{\rho}{2}|\mathbf{u}|^{2}$ is comparable with the pressure, we expect an advantage with this formulation.

The description of other possible algorithms at the numerical level, as well as the analysis of the discretization of the models here presented and a comparative study between the Navier-Stokes formulation here proposed and a more standard one, will be the subject of a forthcoming work.

Acknowledgements. The first and the second authors acknowledge the support by the EU project Haemodel contract HPRN-CT-2002-002670. Partial support by MURST under the project COFIN05 Numerical Modelling for Scientific Computing and Advanced Applications is also acknowledged.

\section{REFERENCES}

[1] C. Begue, C. Conca, F. Murat and O. Pironneau, Les équations de Stokes et de Navier-Stokes avec des conditions aux limites sur la pression, in Nonlinear partial differential equations and their applications, Collège de France Seminar, in Pitman Res. Notes Math. Ser. 181, Longman Sci. Tech., Harlow (1986) 179-264.

[2] H. Beirão da Veiga, On the existence of strong solutions to a coupled fluid-structure evolution problem. J. Math. Fluid Mechanics 6 (2004) 21-52.

[3] C.G. Caro and K.H. Parker, The effect of haemodynamic factors on the arterial wall, in Atherosclerosis - Biology and Clinical Science, A.G. Olsson Ed., Churchill Livingstone, Edinburgh (1987) 183-195. 
[4] P. Causin, J.-F. Gerbeau and F. Nobile, Added-mass effect in the design of partitioned algorithms for fluid-structure problems. Comput. Methods Appl. Mech. Engrg. 194 (2005) 4506-4527.

[5] A. Chambolle, B. Desjardins, M. Esteban and C. Grandmont, Existence of weak solutions for the unsteady interaction of a viscous fluid with an elastic plate. J. Math. Fluid Mech. 7 (2005) 368-404.

[6] P.G. Ciarlet, Mathematical Elasticity. Volume 1: Three Dimensional Elasticity. Elsevier, second edition (2004).

[7] C. Conca, F. Murat and O. Pironneau, The Stokes and Navier-Stokes equations with boundary conditions involving the pressure. Japan J. Math. 20 (1994) 279-318.

[8] D. Coutand and S. Shkoller, The interaction between quasilinear elastodynamics and the Navier-Stokes equations. Arch. Rational Mech. Anal. 179 (2006) 303-352.

[9] L. Formaggia, J.F. Gerbeau, F. Nobile and A. Quarteroni, On the coupling of 3D and 1D Navier-Stokes equations for flow problems in compliant vessels. Comput. Methods Appl. Mech. Engrg. 191 (2001) 561-582.

[10] L. Euler, Principia pro motu sanguinis per arterias determinando. Opera posthima mathematica et physica anno 1844 detecta 2 (1775) 814-823.

[11] M.A. Fernández and M. Moubachir, A Newton method using exact Jacobian for solving fluid-structure coupling. Comput. Struct. 83 (2005) 127-142.

[12] M.A. Fernández, J.-F. Gerbeau and C. Grandmont, A projection semi-implicit scheme for the coupling of an elastic structure with an incompressible fluid. Inter. J. Num. Meth. Eng. 69 (2007) 794-821.

[13] L. Formaggia and A. Veneziani, Reduced and multiscale models for the human cardiovascular system. Lecture notes VKI Lecture Series 2003-07, Brussels (2003).

[14] L. Formaggia, F. Nobile, A. Quarteroni and A. Veneziani, Multiscale modeling of the circulatory system: a preliminary analysis. Comput. Visual. Sci. 2 (1999) 75-83.

[15] L. Formaggia, J.F. Gerbeau, F. Nobile and A. Quarteroni, Numerical treatment of defective boundary conditions for the Navier-Stokes equations. SIAM J. Num. Anal. 40 (2002) 376-401.

[16] L. Formaggia, D. Lamponi, M. Tuveri and A. Veneziani, Numerical modeling of 1D arterial networks coupled with a lumped parameters description of the heart. Comput. Methods Biomech. Biomed. Eng. 9 (2006) 273-288.

[17] L. Formaggia, A. Quarteroni and A. Veneziani, The circulatory system: from case studies to mathematical modelling, in Complex Systems in Biomedicine, A. Quarteroni, L. Formaggia and A. Veneziani Eds., Springer, Milan (2006) 243-287.

[18] V.E. Franke, K.H. Parker, L.Y. Wee, N.M. Fisk and S.J. Sherwin, Time domain computational modelling of 1D arterial networks in monochorionic placentas. ESAIM: M2AN 37 (2003) 557-580.

[19] J.-F. Gerbeau and M. Vidrascu, A quasi-Newton algorithm based on a reduced model for fluid-structure interaction problems in blood flows. ESAIM: M2AN 37 (2003) 631-647.

[20] J.-F. Gerbeau, M. Vidrascu and P. Frey, Fluid-structure interaction in blood flows on geometries coming from medical imaging. Comput. Struct. 83 (2005) 155-165.

[21] F.J.H. Gijsen, E. Allanic, F.N. van de Vosse and J.D. Janssen, The influence of the non-Newtonian properies of blood on the flow in large arteries: unsteady flow in a $90^{\circ}$ curved tube. J. Biomechanics 32 (1999) 705-713.

[22] V. Giraut and P.-A. Raviart, Finite element method fo the Navier-Stokes equations, in Computer Series in Computational Mathematics 5, Springer-Verlag (1986).

[23] J. Gobert, Une inégalité fondamentale de la théorie de l'élasticité. Bull. Soc. Royale Sciences Liège, 31 ${ }^{e}$ année (3-4) (1962) $182-191$.

[24] J. Heywood, R. Rannacher and S. Turek, Artificial boundaries and flux and pressure conditions for the incompressible NavierStokes equations. Int. J. Num. Meth. Fluids 22 (1996) 325-352.

[25] K. Laganà, G. Dubini, F. Migliavaca, R. Pietrabissa, G. Pennati, A. Veneziani and A. Quarteroni Multiscale modelling as a tool to prescribe realistic boundary conditions for the study of surgical procedures. Biorheology 39 (2002) 359-364.

[26] D.A. McDonald, Blood flow in arteries. Edward Arnold Ltd (1990).

[27] A. Moura, The Geometrical Multiscale Modelling of the Cardiovascular System: Coupling 3D and 1D FSI models. Ph.D. thesis, Politecnico di Milano (2007).

[28] R.M. Nerem and J.F. Cornhill, The role of fluid mechanics in artherogenesis. J. Biomech. Eng. 102 (1980) 181-189.

[29] F. Nobile and C. Vergara, An effective fluid-structure interaction formulation for vascular dynamics by generalized Robin conditions. Technical Report 97, MOX (2007).

[30] M.S. Olufsen, C.S. Peskin, W.Y. Kim, E.M. Pedersen, A. Nadim and J. Larsen, Numerical simulation and experimental validation of blood flow in arteries with structured-tree outflow conditions. Ann. Biomed. Eng. 28 (2000) 1281-1299.

[31] T.J. Pedley, The fluid mechanics of large blood vessels. Cambridge University Press (1980).

[32] T.J. Pedley, Mathematical modelling of arterial fluid dynamics. J. Eng. Math. 47 (2003) 419-444.

[33] K. Perktold and G. Rappitsch, Mathematical modeling of local arterial flow and vessel mechanics, in Computational Methods for Fluid Structure Interaction, Pitman Research Notes in Mathematics 306, J. Crolet and R. Ohayon Eds., Harlow, Longman (1994) 230-245.

[34] K. Perktold, M. Resch and H. Florian, Pulsatile non-Newtonian flow characteristics in a three-dimensional human carotid bifurcation model. J. Biomech. Eng. 113 (1991) 464-475. 
[35] A. Quaini and A. Quarteroni, A semi-implicit approach for fluid-structure interaction based on an algebraic fractional step method. Technical Report 90, MOX (2006).

[36] A. Quarteroni, Cardiovascular mathematics, in Proceedings of the International Congress of Mathematicians, Vol. 1, M. SanzSolé, J. Soria, J.L. Varona and J. Vezdeza Eds., European Mathematical Society (2007) 479-512.

[37] A. Quarteroni, M. Tuveri and A. Veneziani, Computational vascular fluid dynamics: problems, models and methods. Comput. Visual. Sci. 2 (2000) 163-197.

[38] A. Quarteroni, S. Ragni and A. Veneziani, Coupling between lumped and distributed models for blood flow problems. Comput. Visual. Sci. 4 (2001) 111-124.

[39] S. Sherwin, L. Formaggia, J. Peiró and V. Franke, Computational modelling of 1D blood flow with variable mechanical properties and its application to the simulation of wave propagation in the human arterial system. Int. J. Num. Meth. Fluids 12 (2002) 48-54.

[40] A. Veneziani and C. Vergara, Flow rate defective boundary conditions in haemodinamics simulations. Int. J. Num. Meth. Fluids 47 (2005) 801-183.

[41] I.E. Vignon-Clementel, C.A. Figueroa, K.E. Jansen and C.A. Taylor, Outflow boundary conditions for three-dimensional finite element modeling of blood flow and pressure in arteries. Comput. Methods Appl. Mech. Engrg. 195 (2006) 3776-3796. 